\title{
Families of periodic horseshoe orbits in the restricted three-body problem
}

\author{
E. Barrabés ${ }^{1}$ and S. Mikkola ${ }^{2}$ \\ ${ }^{1}$ Dept. Informàtica i Matemàtica Aplicada, Universitat de Girona, Avd. LLuís Santaló s/n 17071 Girona, Spain \\ e-mail: barrabes@ima.udg.es \\ 2 Tuorla Observatory, University of Turku, 21500, Piikkiö, Finland \\ e-mail: mikkola@utu.fi
}

Received 17 June 2004 / Accepted 15 November 2004

\begin{abstract}
We compute families of symmetric periodic horseshoe orbits in the restricted three-body problem. Both the planar and three-dimensional cases are considered and several families are found. We describe how these families are organized as well as the behavior along and among the families of parameters such as the Jacobi constant or the eccentricity. We also determine the stability properties of individual orbits along the families. Interestingly, we find stable horseshoe-shaped orbit up to the quite high inclination of $17^{\circ}$.
\end{abstract}

Key words. celestial mechanics

\section{Introduction}

Until recently, horseshoe orbits were mostly theoretical concepts, the only known such motion in the Solar System being the co-orbital pair of Saturnian satellites Janus and Epimetheus. Recently, however, several near Earth asteroids have been found in horseshoe motion with respect to the Earth, examples include Cruithne and 2002 AA29 (see e.g. Wiegert et al. 1997; Connors et al. 2002; Brasser et al. 2004). In Fig. 1 the present orbit of AA29 is illustrated.

Although the horseshoe motion of these asteroids is temporary, it has awaken renewed interest in horseshoe motion and in the theory of co-orbital motion in general (e.g. Christou 2000; Namouni 1999; Namouni et al. 1999).

In the solar system all orbits are very complicated, when studied in detail. However, considerable knowledge can be obtained by studying the restricted three-body problem. (Planetasteroid interactions can often be seen as a restricted three body problem in which the perturbation due to other bodies slowly changes the orbit in the restricted problem phase space.)

The location and stability properties of periodic orbits are basic features of a dynamical system. Therefore it is of interest to find periodic horseshoe orbits, which have been studied rather little, although numerous other periodic orbit studies have been published since the so called Copenhagen problem by Burrau \& Strömgren $(1915,1916,1917)$.

The dynamics of co-orbital satellites have been studied by several authors. One approach is in the framework of the Restricted Three Body Problem, in which one of the satellites is considered as a test particle with zero mass. In this context, both analytical and numerical works have been developed: Dermott \& Murray (1981a,b) and Murray \& Dermott (1999) studied the properties of horseshoe orbits in general and the co-orbital satellites of Saturn in particular using numerical integration and perturbation theory; and Llibre \& Ollé (2001) gave a mechanism for generation of horseshoe orbits. They also performed extensive numerical explorations. Hénon \& Petit (1986) dealt with the co-orbital motion in the context of Hill's problem and Salo \& Yoder (1988) studied the dynamical behavior of several co-orbital bodies moving with the same mean motion around a primary. A different approach considers the full Three Body Problem with two small masses. Spirig \& Waldvogel (1985) study this system as a singular-perturbation problem using matching techniques. Cors \& Hall (2003) investigated the existence of co-orbital motion in terms of two perturbed Kepler problems with small parameters introduced in the equations of the problem.

In this paper we find families of planar and also fully three dimensional symmetric periodic horseshoe orbits in the restricted three-body problem. The objectives of this study are to:

1. find families of planar horseshoe orbits. As a starting point we use the mechanism of generation of horseshoe orbits given by Llibre \& Ollé (2001). We describe how these families are organized and how the Jacobi constant, the eccentricity and the stability parameters vary along and between them;

2. look for bifurcation orbits where the families of spatial orbits are born; 


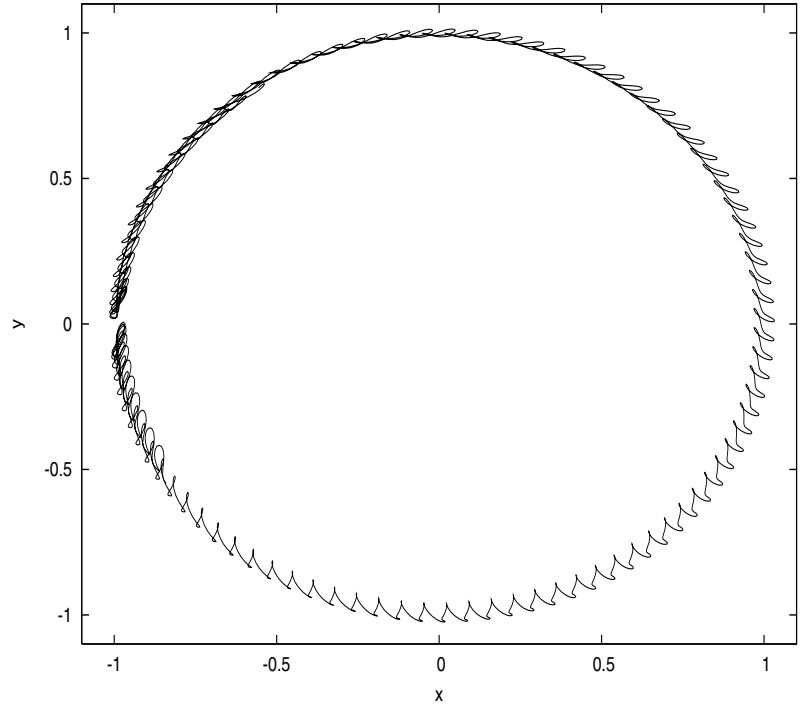

Fig. 1. The present horseshoe orbit of the asteroid 2002 AA29. This is an $x y$-projection in the rotating coordinate system in which the Earth is located at the point $(-1,0)$.

3. compute some families of 3-dimensional horseshoe orbits to describe how the Jacobi constant, the inclination and the stability vary. In particular, we want to see if stable spatial orbits exist and what their inclinations are.

\section{Basic aspects}

First we briefly review the basic mathematics of the Restricted Three Body Problem (RTBP).

Let us consider two bodies $S$ and $E$, called primaries, in an inertial reference system $(O X Y Z$, also called sidereal) describing circular orbits around their center of mass, and a massless particle $P$ which moves under the gravitational attraction of the primaries. The motion of $P$ does not affect the primaries. The description of the motion of the third mass $P$ is known as the circular restricted three body problem. In suitable units the gravitational constant and the distance between the primaries both equal 1. The bodies $S$ and $E$ have masses $1-\mu$ and $\mu$ respectively, with $\mu \in[0,1]$, and the period of their circular motion is $2 \pi$ time units. In the present work we consider only small values of $\mu$.

When the orbit of $P$ is confined in the $Z=0$ plane it is said to be planar, otherwise it is a spatial orbit.

We consider the motions in a rotating reference system (oxyz, called synodical), where the primaries $S$ and $E$ are fixed in the $x$-axis at positions $\mu$ and $\mu-1$, respectively. The equations of motion of the third body in this system are

$\ddot{x}-2 \dot{y}=\frac{\partial \Omega}{\partial x}, \quad \ddot{y}+2 \dot{x}=\frac{\partial \Omega}{\partial y}, \quad \ddot{z}=\frac{\partial \Omega}{\partial z}$

where

$\Omega(x, y, z)=\frac{1}{2}\left(x^{2}+y^{2}\right)+\frac{1-\mu}{r_{1}}+\frac{\mu}{r_{2}}+\frac{1}{2} \mu(1-\mu)$,

and $r_{1}^{2}=(x-\mu)^{2}+y^{2}+z^{2}, r_{2}=(x-\mu+1)^{2}+y^{2}+z^{2}$ are the distances from the third body to $S$ and $E$ respectively. These
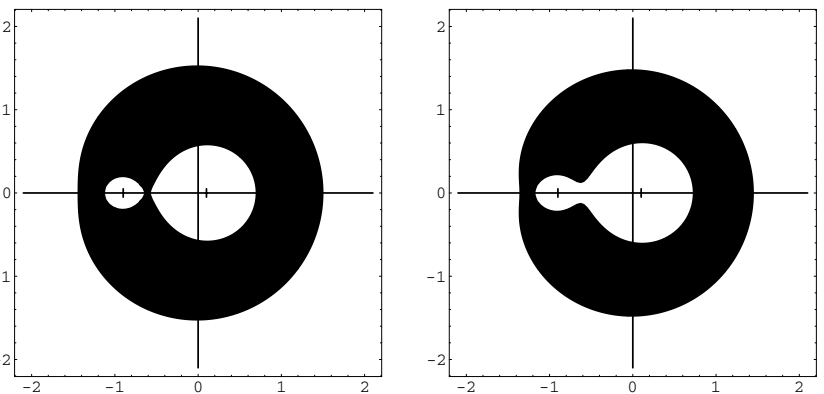

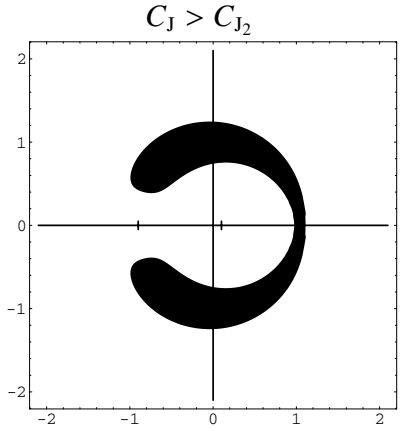

$C_{\mathrm{J}_{3}}<C_{\mathrm{J}}<C_{\mathrm{J}_{1}}$

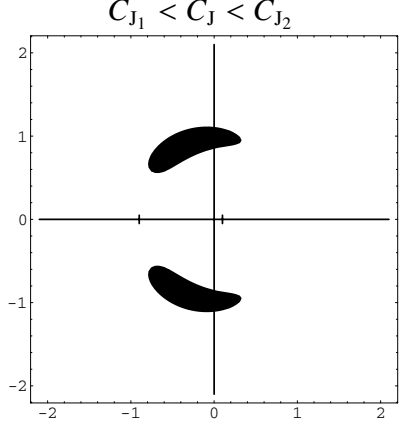

$3<C_{\mathrm{J}}<C_{\mathrm{J}_{3}}$
Fig. 2. Zero velocity curves (the intersection of the zero velocity surfaces with the $z=0$ plane) for $\mu>0$. The motion is forbidden in the filled areas. The tick marks on the horizontal axis show the position of the primaries.

equations have 5 equilibrium points: the collinear points $L_{1}, L_{2}$ and $L_{3}$ and the equilateral ones, $L_{4}$ and $L_{5}$. These are called the Lagrangian points. Furthermore, the Eqs. (1) have the first integral

$\dot{x}^{2}+\dot{y}^{2}+\dot{z}^{2}=2 \Omega(x, y, z)-C_{\mathrm{J}}$,

where $C_{\mathrm{J}}$ is called the Jacobi constant. From now on, we will denote the value of the Jacobi constant at the equilibrium point $L_{i}$ by $C_{\mathrm{J}_{i}}$, for $i=1, \ldots, 5$.

As it is well known, the Hill's regions, where the motion of the third body is possible, are bounded by the zero velocity surfaces $(z v s)$ given by the equation

$C_{\mathrm{J}}=2 \Omega(x, y, z)=x^{2}+y^{2}+2 \frac{1-\mu}{r_{1}}+2 \frac{\mu}{r_{2}}+\mu(1-\mu)$.

For a fixed value of $\mu$, the shape of these surfaces varies with $C_{\mathrm{J}}$ (see, for instance, Szebehely 1967). For large values of the Jacobi constant, $C_{\mathrm{J}}>C_{\mathrm{J}_{2}}$, the regions where the motion is possible are not connected, while for $C_{\mathrm{J}}<3$ the surfaces do not intersect the plane $z=0$ and planar motion is possible everywhere. The intersection of the zero velocity surfaces with the $z=0$ plane (the zero velocity curves) is shown in Fig. 2. As we can see, for planar orbits, the natural range for horseshoe periodic orbits is $C_{\mathrm{J}}<C_{\mathrm{J}_{1}}$ : for $C_{\mathrm{J}} \in\left(C_{\mathrm{J}_{3}}, C_{\mathrm{J}_{1}}\right)$ the zero velocity curve is also horseshoe shaped. In the next section, we look for periodic horseshoe orbits in this range of values of the Jacobi constant; we find that the orbits are embedded in 
Table 1. Position and Jacobi constant for the collinear equilibrium points $L_{i}, i=1,2,3$, and values of $\mu$ indicated. See the text for details.

\begin{tabular}{ccc}
\hline \hline$\mu=10^{-4}$ & $x_{i}$ & $C_{\mathrm{J}_{i}}$ \\
\hline$L_{1}$ & -1.0324251917 & 3.008955890917 \\
$L_{2}$ & -0.9680652061 & 3.009089235145 \\
$L_{3}$ & 1.0000416667 & 3.000199989791 \\
\hline$\mu=0.304018792 \times 10^{-5}$ & $x_{i}$ & $C_{\mathrm{J}_{i}}$ \\
\hline$L_{1}$ & -1.010074939199 & 3.000896881934 \\
$L_{2}$ & -0.989986240081 & 3.000900935559 \\
$L_{3}$ & 1.000001266745 & 3.000006080366 \\
\hline
\end{tabular}

families which continue for $C_{\mathrm{J}}<C_{\mathrm{J}_{3}}$. The collinear equilibrium points are situated at $\left(x_{i}, 0,0\right)$, for $i=1,2,3$, where

$$
\begin{aligned}
& x_{1}=-1-\left(\frac{\mu}{3}\right)^{1 / 3}-\frac{1}{3}\left(\frac{\mu}{3}\right)^{2 / 3}+\frac{28}{9}\left(\frac{\mu}{3}\right)+O\left(\mu^{4 / 3}\right), \\
& x_{2}=-1+\left(\frac{\mu}{3}\right)^{1 / 3}-\frac{1}{3}\left(\frac{\mu}{3}\right)^{2 / 3}+\frac{26}{9}\left(\frac{\mu}{3}\right)+O\left(\mu^{4 / 3}\right), \\
& x_{3}=1+\frac{5}{12} \mu+O\left(\mu^{3}\right)
\end{aligned}
$$

(see, for instance, Szebehely 1967). Using these expressions and Eq. (2), the values of the Jacobi constant at the equilibrium points are

$$
\begin{aligned}
& C_{\mathrm{J}_{1}}=3+9\left(\frac{\mu}{3}\right)^{2 / 3}-11\left(\frac{\mu}{3}\right)+O\left(\mu^{4 / 3}\right), \\
& C_{\mathrm{J}_{2}}=3+9\left(\frac{\mu}{3}\right)^{2 / 3}-7\left(\frac{\mu}{3}\right)+O\left(\mu^{4 / 3}\right), \\
& C_{\mathrm{J}_{3}}=3+2 \mu-\frac{49}{48} \mu^{2}+O\left(\mu^{3}\right) .
\end{aligned}
$$

In Table 1 the values of $x_{i}$ and $C_{\mathrm{J}_{i}}, i=1,2,3$, for $\mu=10^{-4}$ and $\mu=0.304018792 \times 10^{-5}$ (Sun-(Earth+Moon) mass ratio) are shown.

In order to deal with a small value of $\mu$, but big enough to show the behavior of the families, the explorations presented in this work are done for $\mu=10^{-4}$. Whenever a different value of $\mu$ is taken for comparison, it will be specified. For greater clarity, we include two dots in the plots of orbits where the primaries are located.

- We integrate the equations of motion and the variational equations using a Taylor-series method for ordinary differential equations. We use the software package for the numerical integration of Ordinary Differential Equations provided by Jorba \& Zou (preprint).

- We find the periodic orbits using the Newton-Raphson method to correct the initial values until periodicity with a tolerance of $10^{-12}$ is achieved.

\section{Planar horseshoe orbits}

In this section, we suppose that the motion of the third body is planar, so $z=\dot{z}=0$ for all $t$. For simplicity, we denote $\boldsymbol{r}=(x, y)$ and $\dot{\boldsymbol{r}}=(\dot{x}, \dot{y})$. (Later we will re-introduce the $z$ and $\dot{z}$ components.)

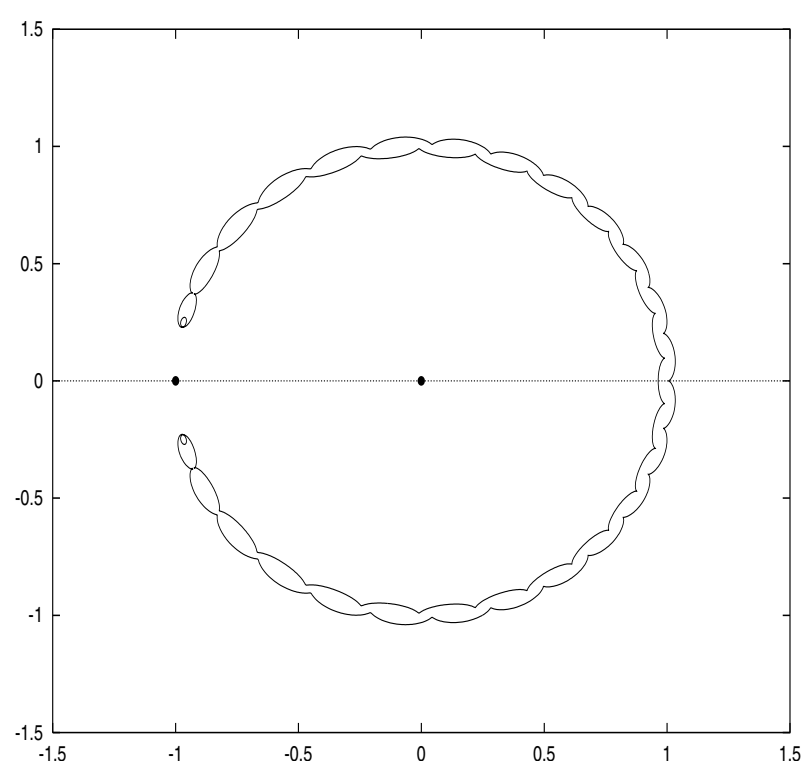

Fig. 3. Planar symmetric horseshoe orbit with only two crossings with the synodical $x$ axis.

According to Llibre \& Ollé (2001), a planar symmetric periodic horseshoe orbit is a planar periodic solution in which the particle follows a path which surrounds only the equilibrium points $L_{3}, L_{4}$ and $L_{5}$ and has only two orthogonal crossings with the $x$ axis. This definition describes an orbit with a horseshoe shape resembling the zero velocity curves ( $z v c$ from now on) for $C_{\mathrm{J}} \in\left(C_{\mathrm{J}_{3}}, C_{\mathrm{J}_{1}}\right)$ (see Fig. 2) and two orthogonal crossings with the horizontal synodical axis near $L_{3}$. In order to follow families of periodic horseshoe orbits, we begin with horseshoe orbits with a value of $C_{\mathrm{J}} \in\left(C_{\mathrm{J}_{3}}, C_{\mathrm{J}_{1}}\right)$ and initial conditions close to the zero velocity curve, so their shape coincides with this definition (see, for example, Fig. 3). But when we continue the families, we find that the number of crossings with the $x$ axis increases. The orbits can have more intersections near $L_{3}$ due to the increase of loops (see Fig. 5). It is also possible to find orbits that briefly enter the upper half plane but go back after a short time into the lower half plane and vice versa (see Fig. 4). In the first case, the horseshoe shape is preserved, but not in the second one. Nevertheless, in the present work we consider all of them as horseshoe orbits.

We restrict our attention to symmetric periodic orbits. Due to the fact that the equations of motion (1) of the planar RTBP are invariant by

$(t, x, y, \dot{x}, \dot{y}) \longrightarrow(-t, x,-y,-\dot{x}, \dot{y})$

if a solution of these equations has two orthogonal crossings with the $x$ axis, then it is symmetric with respect to this axis. Thus, for a symmetric periodic orbit we suppose that one orthogonal crossing occurs at $t=0$ and the other one at $t=T / 2$, where $T$ is the period of the orbit. The initial conditions (at $t=0)$ will be $\boldsymbol{r}_{0}=\left(x_{0}, 0\right)$ and $\dot{\boldsymbol{r}}_{0}=\left(0, \dot{y}_{0}\right)$ and the final condition (at $t=T / 2)$ will be $\boldsymbol{r}_{\mathrm{f}}=\left(x_{\mathrm{f}}, 0\right)$ and $\dot{\boldsymbol{r}}_{\mathrm{f}}=\left(0, \dot{y}_{\mathrm{f}}\right)$. Notice that for horseshoe orbits, the initial and final positions are near $L_{3}$.

Finally, due to the behavior of the zero velocity curves, we look for periodic horseshoe orbits that satisfy $C_{\mathrm{J}}<C_{\mathrm{J}_{1}}$. 

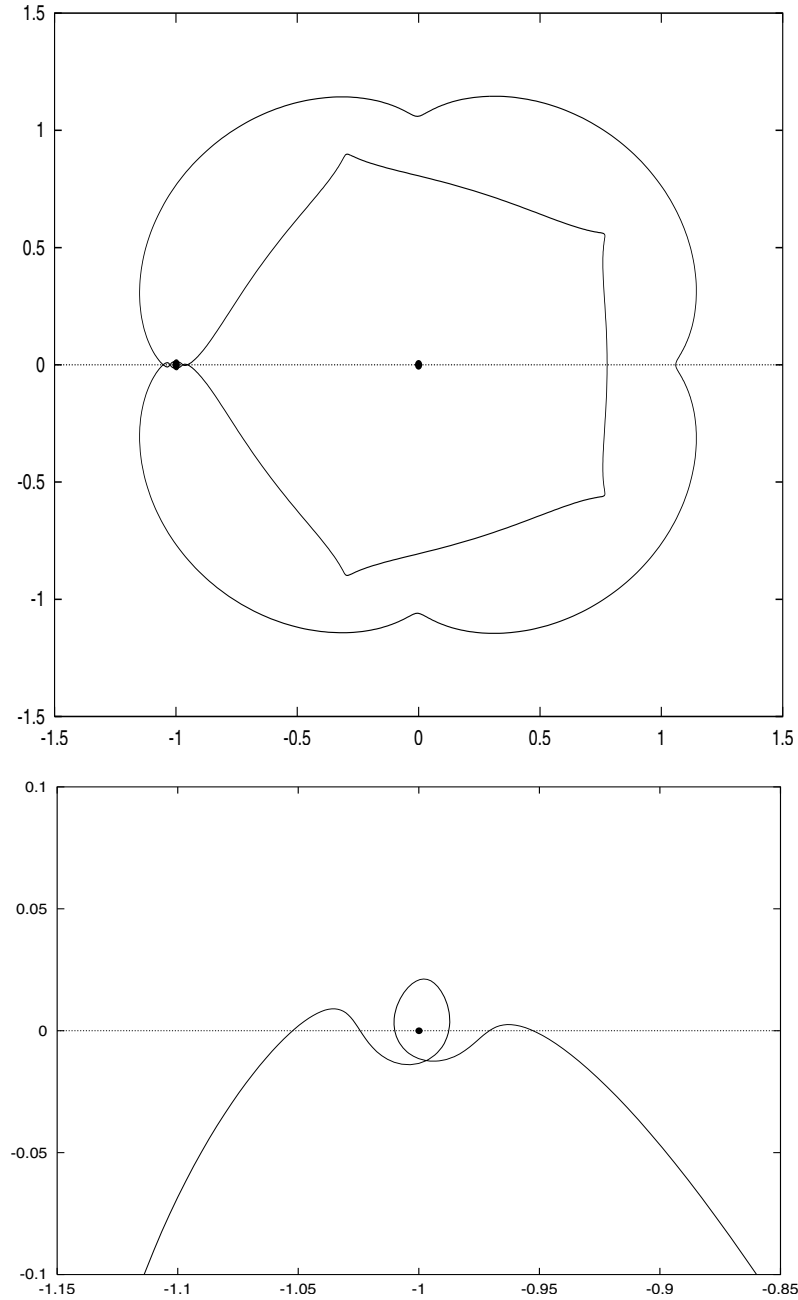

Fig. 4. Planar symmetric horseshoe orbit with 14 crossings with the synodical $x$ axis and a detail of the passages near the small primary. On the top, one period is plotted and on the bottom just a half period.

\subsection{Finding planar periodic horseshoe orbits}

Next, we describe the steps followed to obtain families of periodic horseshoes. First, we follow the same strategy as in Llibre \& Ollé (2001), looking for horseshoes with the same value of $C_{\mathrm{J}}$ less than $C_{\mathrm{J}_{1}}$. As Llibre \& Ollé pointed out in their work, many periodic horseshoe orbits can be found for small $\mu>0$. These orbits, far from the small primary, can be approximated by a pair of orbits of the two-body problem (RTBP for $\mu=0$ ): a retrograde one, which is the outer approximation (corresponding to the outer part of the orbit) and a direct one, which is the inner approximation (corresponding to the inner part of the orbit). For $\mu>0$, the effect of the small primary makes the third body to go back, passing from the outer part of the orbit to the inner, or vice versa. The outer and inner part of the orbit can be easily observed in the behavior of the eccentricity and the semi-major axis of the orbits. The values of these parameters change at every near(est) passage of the third body and the small primary (Fig. 6).

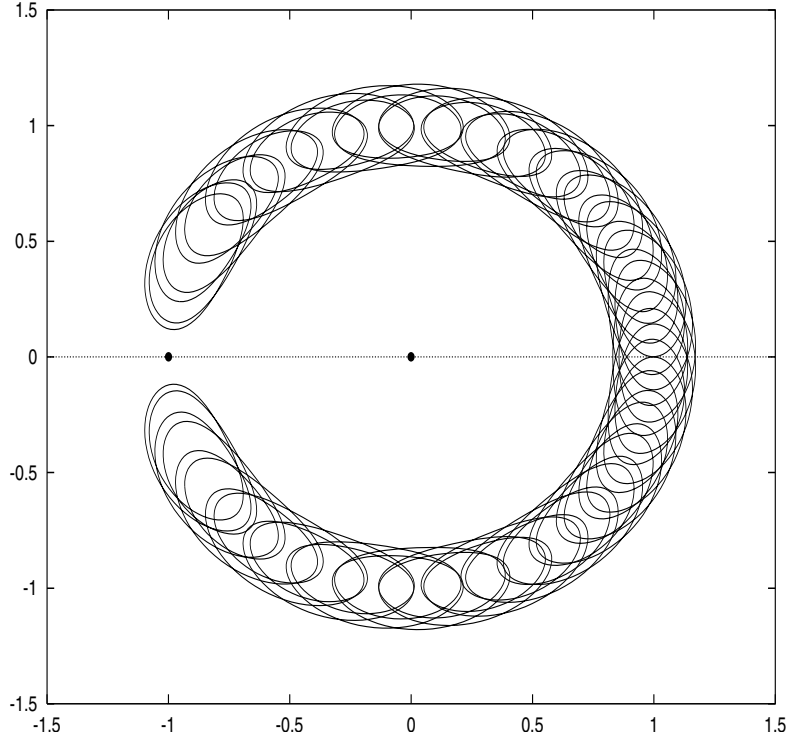

Fig. 5. Planar symmetric horseshoe orbit with 16 crossings with the synodical $x$ axis near $L_{3}$.
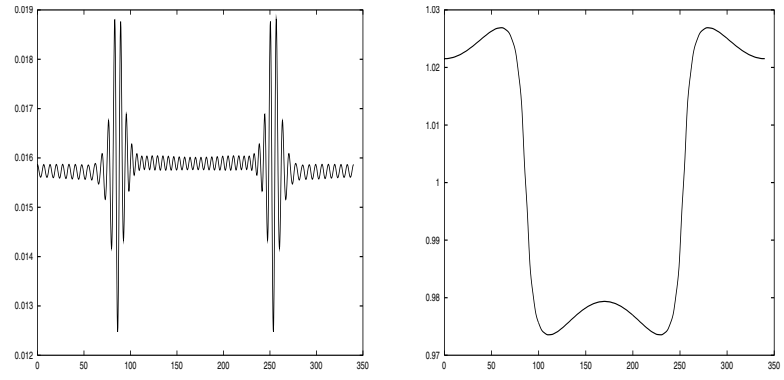

Fig. 6. Behaviour of the eccentricity (left) and semi-major axis (right) of the orbit shown in Fig. 3. Both parameters change at each approach to the small primary.

For a fixed $C_{\mathrm{J}} \in\left(C_{\mathrm{J}_{3}}, C_{\mathrm{J}_{1}}\right)$, the procedure is (for more details see Llibre \& Ollé 2001):

1. we look for the intersection of the $z v c$ with the horizontal axis at the right side of $L_{3}$. We start with a value of $x_{0}$ close to and at the right side of this intersection and with a negative initial $\dot{y}_{0}$ given by Eq. (2);

2. we integrate the equations of motion until the next crossing with the $x$ axis near $L_{3}$ (rejecting the orbits without a horseshoe shape), so the final point will be $\left(x_{\mathrm{f}}, 0, \dot{x}_{\mathrm{f}}, \dot{y}_{\mathrm{f}}\right)$. We are looking for the points $x_{0}$ were the function $\dot{x}_{\mathrm{f}}=$ $\dot{x}_{\mathrm{f}}\left(t_{\mathrm{f}}, x_{0}, \dot{y}_{0}\right)=0$, so we increase $x_{0}$ and continue until a change in the sign of $\dot{x}_{\mathrm{f}}$ is detected. Then, between the last two values of $x_{0}$ there must exist one value which corresponds to the initial conditions of a symmetric periodic orbit, the exact value of which we find using the secant method.

The function $\dot{x}_{\mathrm{f}}\left(t_{\mathrm{f}}, x_{0}, \dot{y}_{0}\right)$ as a function of $x_{0}$ for two fixed values of $C_{\mathrm{J}}$ is shown in Fig. 7.

As expected, many horseshoe orbits can be found. It must be taken into account that the number of intersections with the section $y=0$ can vary from one periodic orbit to the next. 

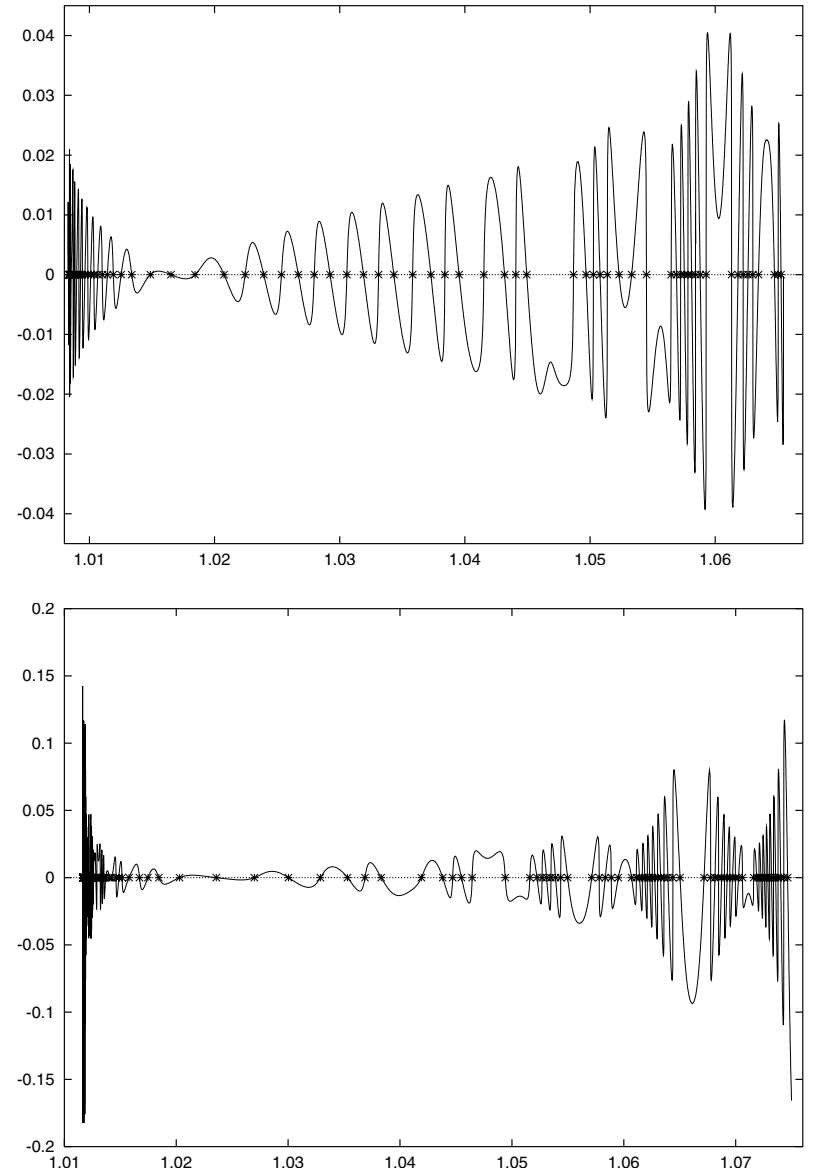

Fig. 7. $\dot{x}_{\mathrm{f}}$ as a function of $x_{0}$ for the the values of the Jacobi constant $C_{\mathrm{J}}=3.0004$ on the top and $C_{\mathrm{J}}=3.0006$ on the bottom. See the text for more details.

Moreover, we can make several observations from the results obtained:

1. The function $\dot{x}_{\mathrm{f}}$ oscillates around the horizontal axis, with variations in its amplitude. The zeros of $\dot{x}_{\mathrm{f}}$ are not distributed uniformly with respect to $x_{0}$. They are more concentrated near the intersection of the $z v c$ with the $x$ axis than far from it.

2. The number of intersections of the periodic orbits with the synodical horizontal axis increases both for values of their initial condition $x_{0}$ near the $z v c$ and far from it, while for the other values of $x_{0}$, the horseshoe orbits obtained have only two crossings with the $x$ axis. Furthermore, when $x_{0}$ approaches the $z v c$ or goes far from it, several zones (a range of values of $x_{0}$ ) appear where horseshoe orbits do not exist. This behavior depends strongly on the value of $C_{\mathrm{J}}$ : for values near $C_{\mathrm{J}_{1}}$ the behavior of $\dot{x}_{\mathrm{f}}$ is more irregular. As we are looking for a method to obtain initial periodic orbits as starting point from which to compute the families, we have not explored all the possible values of $x_{0}$. So we have represented the function only for a certain range of values.

3. For each periodic orbit, we compute the planar stability parameter $s_{1}$, given by $s_{1}=2-\operatorname{tr}\left(M_{\mathrm{p}}\right)$, where $M_{\mathrm{p}}$ is the monodromy matrix (obtained from the integration of the variational equations with the equations of motion) at $t=T$ (the

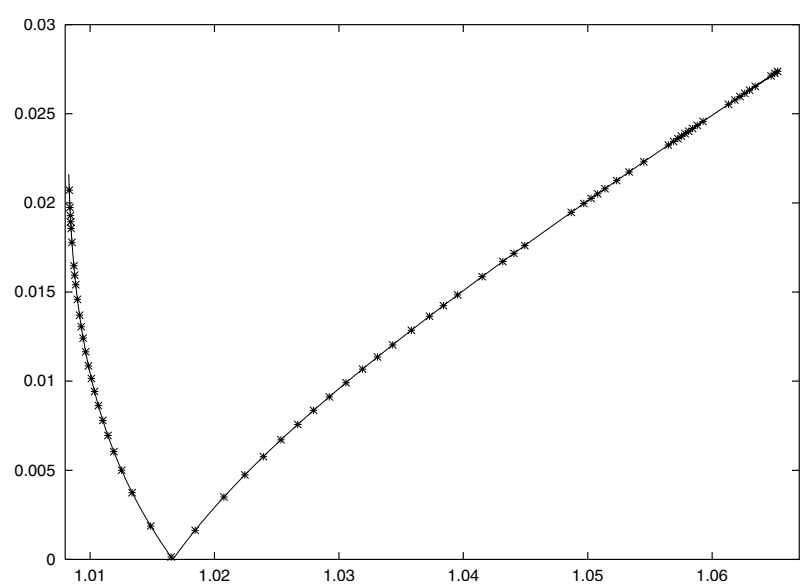

Fig. 8. Eccentricity of the orbits computed for $C_{\mathrm{J}}=3.0004 . x_{0}$ is on the horizontal axis. The points marked correspond to the points $\dot{x}_{\mathrm{f}}=0$ (periodic orbits) in Fig. 7.

period of the orbit). We observe that there are (linearly) stable $\left(\left|s_{1}\right|<2\right)$ and unstable orbits, and that the unstable ones have mostly initial condition $x_{0}$ very near or very far from the $z v c$.

4. As we know, the eccentricity of a horseshoe orbit is not constant. However, the eccentricity at the initial conditions can be used without loss of generality. This value of $e$ corresponds to the eccentricity of the outer approximation twobody orbit. Thus, for each orbit, we compute the eccentricity by

$e=\left|1-x_{0}\left(x_{0}+\dot{y}_{0}\right)^{2}\right|$.

In Fig. 8 the behavior of the eccentricity as a function of $x_{0}$ for a fixed value of $C_{\mathrm{J}}$ is shown. As long as the eccentricity is small, the number of intersections with the synodical horizontal axis is two, whereas, when the eccentricity increases, new loops can appear and the number of intersections increases as well. Moreover, as we can see in Fig. 8, there is a minimum $(e \approx 0)$ coinciding with a periodic orbit. This horseshoe orbit corresponds to a zero of the zone, where the function $\dot{x}_{\mathrm{f}}$ has low amplitude, and it is planar stable. This is the orbit that we choose as the starting point in the computation of a family of periodic orbits.

\subsection{Families of planar periodic horseshoe orbits}

As is well known, periodic orbits are not isolated but form oneparameter families. A family of planar symmetric periodic orbits with initial conditions $\left(x_{0}, 0,0, \dot{y}_{0}\right)$ is defined implicitly by the equation

$\dot{x}\left(T / 2, x_{0}, \dot{y}_{0}\right)=0$,

where $T=T\left(x_{0}, \dot{y}_{0}\right)$ is given by the section $y\left(T / 2, x_{0}, \dot{y}_{0}\right)=0$. In order to compute a family of periodic orbits we use a predictor-corrector method:

1. Once we have obtained a periodic orbit, we predict the next one moving along the tangent of the Eq. (5). 
2. We correct the initial conditions of the predicted orbit. The Eq. (5) has two unknowns, thus two strategies can be followed:

- one may fix the value of the Jacobi constant and use Newton's method;

- one may ask for minimum variation of the initial conditions, such that the new initial conditions verify Eq. (5). In this case we have a constrained extrema problem.

In Gómez \& Mondelo (2001) a method for the refinement and continuation of periodic orbits is given in detail. To begin with this method, it is necessary to have an initial periodic orbit. The horseshoe orbit computed in the previous section with $\left|s_{1}\right|<2$ and minimum value of $e$, for different values of $C_{\mathrm{J}}$, is chosen for this purpose.

The families followed are shown in Fig. 9 for different ranges of values of $C_{\mathrm{J}}$. They are represented in the $\left(x_{0}, C_{\mathrm{J}}\right)$ plane. In these representations, the zero velocity curve is also plotted. Its equation in the $\left(x_{0}, C_{\mathrm{J}}\right)$ plane is given by

$C_{\mathrm{J}}=\frac{2(1-\mu)}{x_{0}-\mu}+\frac{2 \mu}{x_{0}+1-\mu}+\mu(1-\mu)+x_{0}^{2}$,

which can be obtained substituting $y=z=0$ in Eq. (3). As we can see, the families are bounded on the left by the zero velocity curve.

Moreover, we compute the values of $x_{0}$ and $C_{\mathrm{J}}$ for which the outer approximation with the same initial conditions has an eccentricity of zero. If we make $e=0$ in Eq. (4), then we can write

$\dot{y}_{0}=-x_{0}+\frac{1}{\sqrt{x_{0}}}$,

and substituting this expression in Eq. (2), we obtain

$C_{\mathrm{J}}=\frac{2(1-\mu)}{x_{0}-\mu}+\frac{2 \mu}{x_{0}+1-\mu}+\mu(1-\mu)+2 \sqrt{x_{0}}-\frac{1}{x_{0}}$.

This curve (the skeleton) is the dashed line in the figures. For $\mu=0$, Eq. (7) represents two families of the circular Kepler orbits (denoted by l-i in the literature) which give the outer and inner approximations of the corresponding horseshoe orbits.

From our results we observe the following:

1. the skeleton gives a first approach to find horseshoe orbits for $C_{\mathrm{J}}$ values that are not too big. The approximation is better when $\mu$ decreases;

2. a priori, the maximum value of the Jacobi constant for which horseshoe orbits exist is $C_{\mathrm{J}_{1}}$, whose value is 3.008955890917 for $\mu=10^{-4}$. As we can see in Fig. 9, the bigger the value of $C_{\mathrm{J}}$ the fewer families we find. $C_{\mathrm{J}}=3.008183416975$ is the maximum value of the Jacobi constant for which we find a periodic orbit (Fig. 4);

3 . although in the previous section we dealt with initial conditions such that $\dot{y}_{0}<0$, when we continue the families we find horseshoe orbits with $\dot{y}_{0}>0$. This happens, for example, when one family reaches the $z v c$ : the sign of the velocity changes;

4. for each family, there exists a maximum for the Jacobi constant, $C_{\mathrm{Jm}}$. If the Jacobi constant is not too big, the families are organized around the skeleton in such a way that a
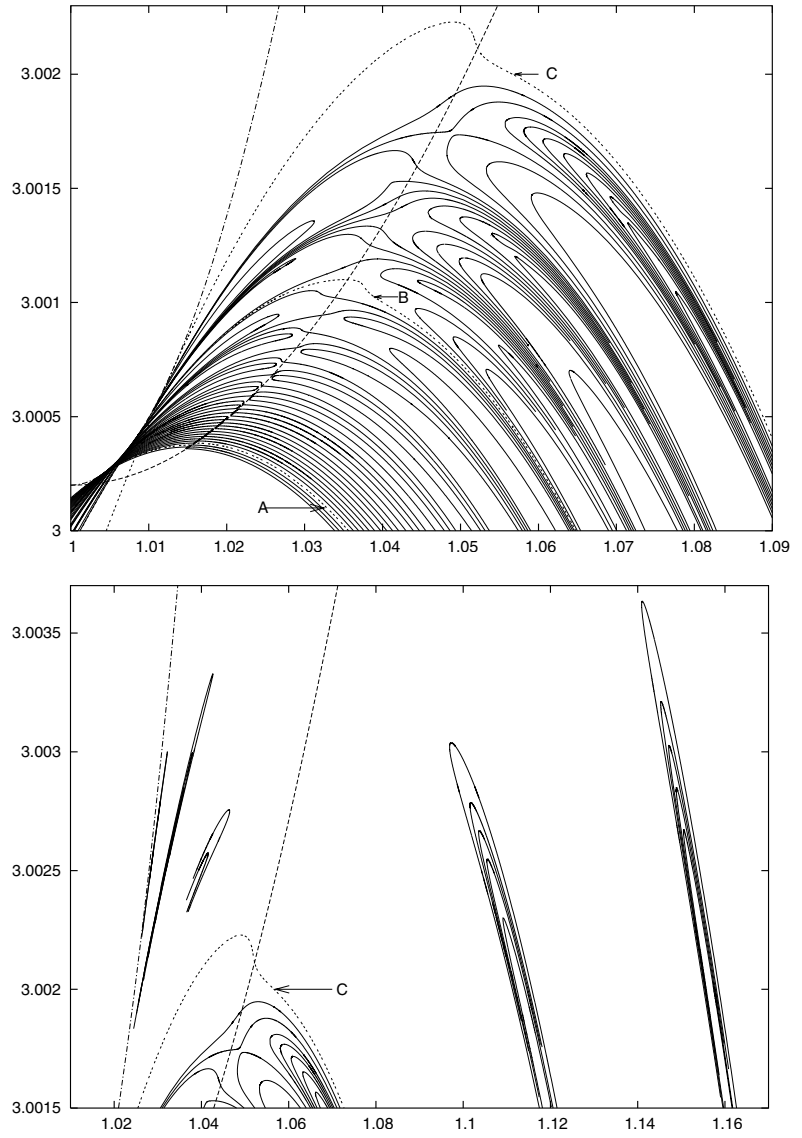

Fig. 9. Families of planar and symmetric horseshoe orbits in the $\left(x_{0}, C_{\mathrm{J}}\right)$ plane for $\mu=10^{-4}$ and different range of values for $C_{\mathrm{J}}$. The dashed-dotted line on the right represents the zero velocity curve (6). The dashed line crossing all the families is the skeleton given by Eq. (7). Three families, marked A, B and C, have been chosen to illustrate the evolution of the orbits along and between the families. See the text for more details. a) Families with $C_{\mathrm{Jm}} \leq 3.0022$; b) families with $C_{\mathrm{Jm}} \geq 3.0015$.

structure consisting of 4 families is repeated (see Fig. 10). Nevertheless, for large enough $C_{\mathrm{Jm}}$ this structure disappears;

5. the period of the periodic orbits varies inside the families and between them. The behavior of the period along the families is illustrated in Fig. 11. The smaller the value of $C_{\mathrm{Jm}}$ of a family, the longer the period of the orbits. For families with smaller values of $C_{\mathrm{Jm}}$ a structure exists (see Fig. $11 \mathrm{~b}$ ) while as the value of $C_{\mathrm{Jm}}$ increases, this structure disappears (see Fig. 11c). Two orbits from different families typically have different periods, while pairs of orbits from different families with the same period can be found (for values of $C_{\mathrm{Jm}}$ greater than a certain value);

6. in each family, as $C_{\mathrm{J}}$ decreases the eccentricity (given by Eq. (4)) increases and so does the number of loops in the rotating frame. See Fig. 5;

7. for each orbit, we compute the minimum distance $d_{\mathrm{p}}$ of the third body to the small primary as well. Within each family, $d_{\mathrm{p}}$ decreases as $C_{\mathrm{J}}$ decreases, except for a small range of values in a neighborhood of $C_{\mathrm{J}}=C_{\mathrm{Jm}}$. Between families, the bigger the value of $C_{\mathrm{Jm}}$, the smaller the minimum 


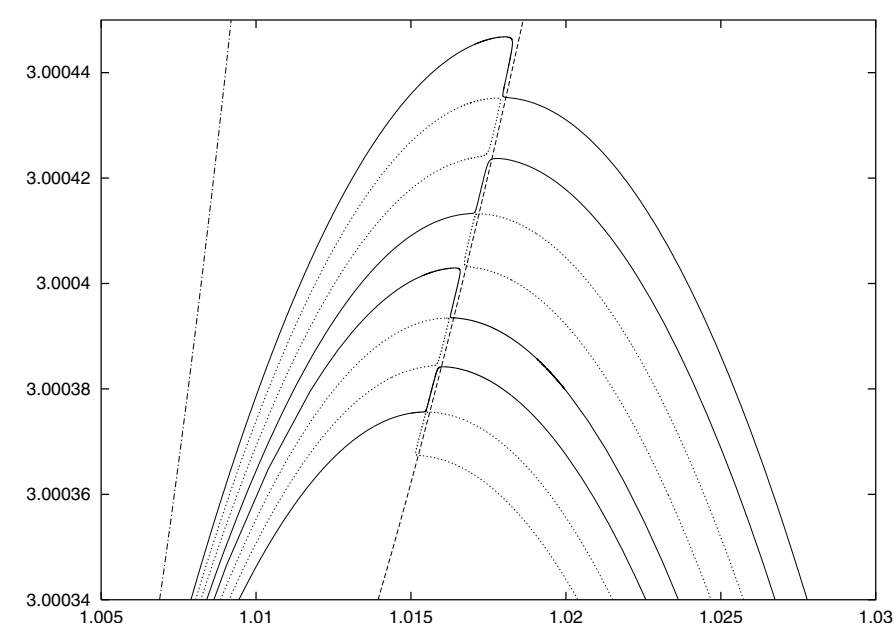

Fig. 10. Details around the skeleton of some families for $\mu=10^{-4}$. Apparently, the structure repeats after every four families.

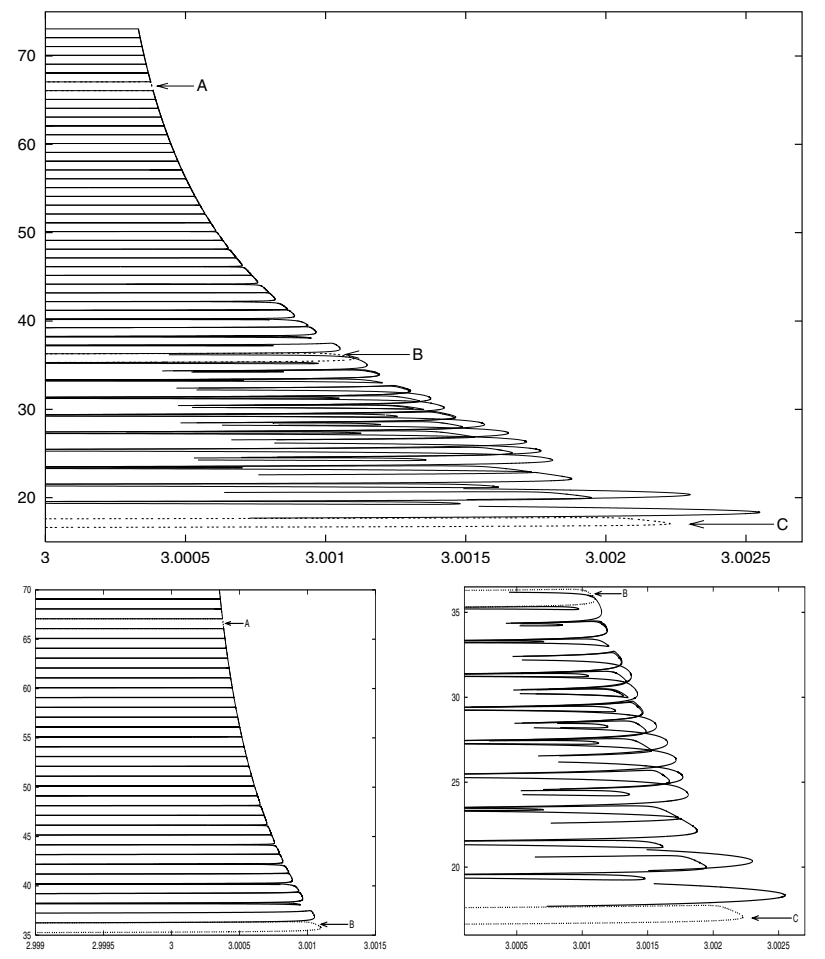

Fig. 11. Behaviour of the period ( $T$ in years) of the orbits on each family with respect to $C_{\mathrm{J}}$. The bigger the value of $C_{\mathrm{Jm}}$, the smaller the period of the orbits. Families A, B and C are the same of that of Fig. 9. a) The families shown are the same as in Fig. 9a; b) families with $T \geq 35$ years; c) families with $T \leq 36.5$ years.

distance. All the orbits computed for values of the Jacobi constant greater than a certain value enter a neighborhood of the small primary. This fact is explained by the Jacobi constant. If an orbit does not reach a neighborhood of the small primary, say, $r_{2}>\alpha\left(\frac{\mu}{3}\right)^{1 / 3}$, then from Eq. (2) we obtain

$C_{J} \leq 3+\frac{6}{\alpha}\left(\frac{\mu}{3}\right)^{2 / 3}$.

See Fig. 12;

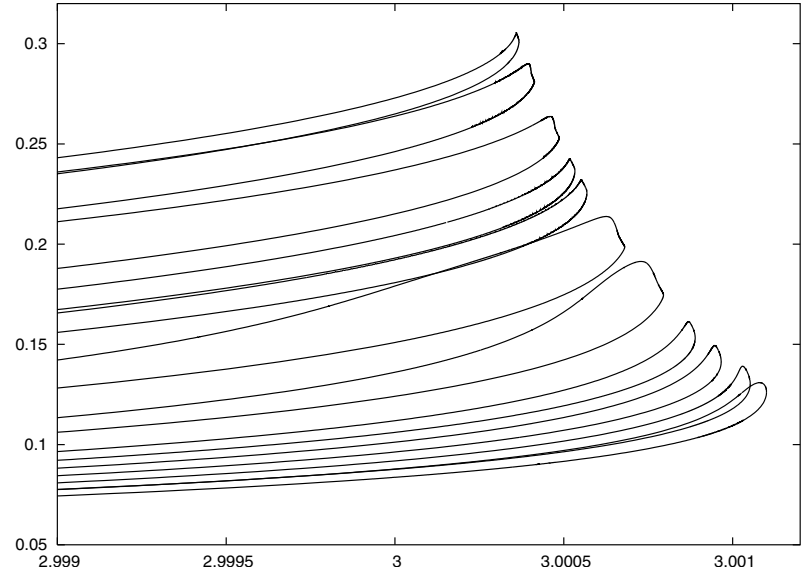

Fig. 12. Behaviour of the minimum distance $d_{\mathrm{p}}$ to the small primary on each family with respect to $C_{\mathrm{J}}$. For greater clarity, just a few families are plotted.

8. orbits that briefly enter the upper half plane are found for values of $C_{\mathrm{Jm}} \geq 3.00499$. These families are not shown in Fig. 9, which contains only pure horseshoe orbits (orbits with a horseshoe shape);

9. it is known that, either a family of periodic orbits closes upon itself or it is an open family. In the latter case, the family has, in both directions, a natural termination in which the dimension of the orbit, the Jacobi constant or the period grow without limit (see Hénon 1997, p. 10). Moreover, given a family we observe that when $C_{\mathrm{J}}$ decreases $\left|s_{1}\right|$ increases. We follow the families only up to a certain value of $\left|s_{1}\right|$.

In order to show the evolution of the shape of the orbits along one family and the difference between different families, we have chosen three families labeled A, B and C in Figs. 9 and 11 . The approximate values of $C_{\mathrm{Jm}}$ in each family are 3.0003841802 (A), 3.0011003259 (B) and 3.0022285012 (C). Figures 13-15 illustrate some of the orbits in each family (A, $\mathrm{B}$ and $\mathrm{C}$ respectively). In each figure, orbits from left to right and top to bottom correspond to increasing values of the initial $x_{0}$. Table 2 contains the Jacobi constant $C_{\mathrm{J}}$, the period $T$ in years, the initial conditions $x_{0}, \dot{y}_{0}$ and the stability parameter $s_{1}$ of these orbits. Both the orbit with the maximum value of the Jacobi constant $C_{\mathrm{Jm}}$ and the orbit with the minimum value of the eccentricity of each family are included in the examples shown.

Along both branches of each family (this is, as $C_{\mathrm{J}}$ decreases), the number of loops increases (corresponding to an increase of the eccentricity) as does the width of the orbits. Moreover, the larger the value of $C_{\mathrm{Jm}}$ of one family, the larger the width of the orbits of this family and the shorter the minimum distance to the small primary. This is because the horseshoe orbits with $C_{J}>3$ surround the zero velocity curve, the width of which increases with the value of the Jacobi constant.

Summarizing, the resemblances between families are:

- there exists one orbit inside each family with the maximum value of the Jacobi constant, $C_{\mathrm{Jm}}$. Starting at this orbit, the family has two branches along which $C_{\mathrm{J}}$ decreases; 
(1)

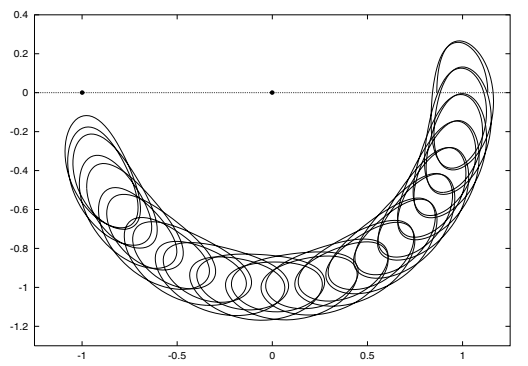

(4)

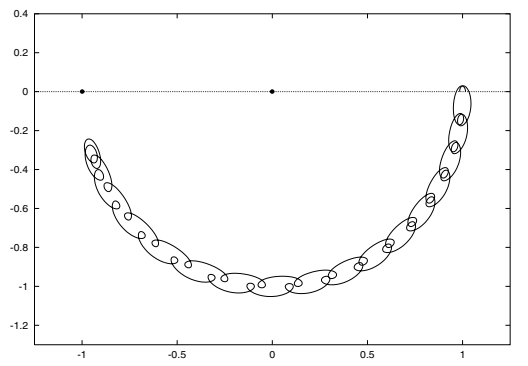

(7)

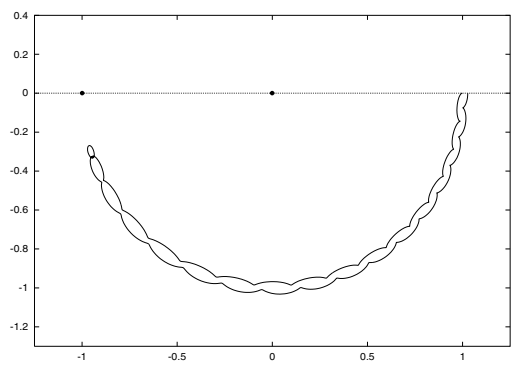

(10)

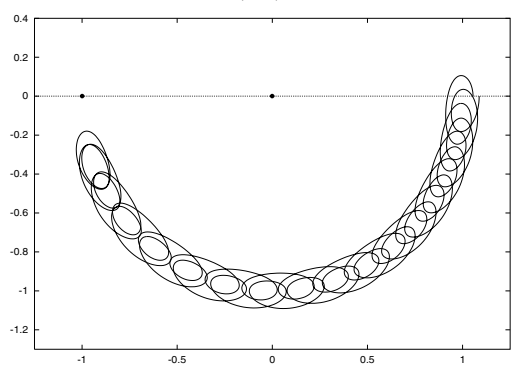

(2)

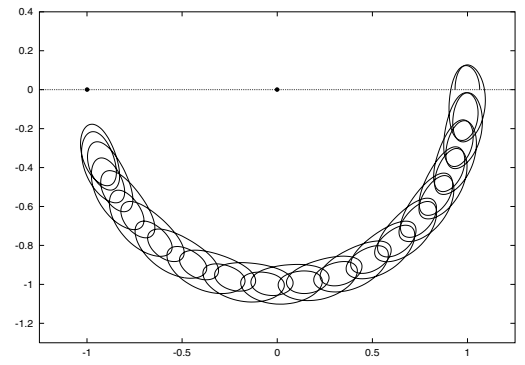

(5)

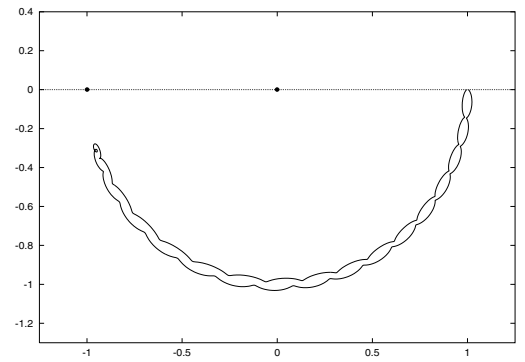

(8)

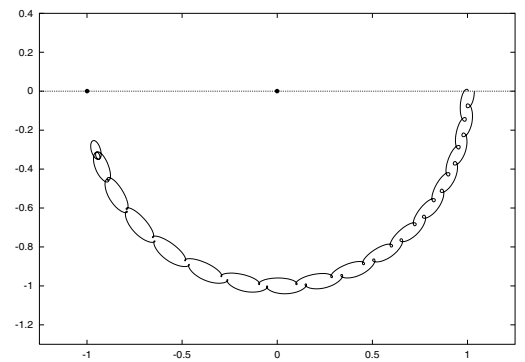

(11)

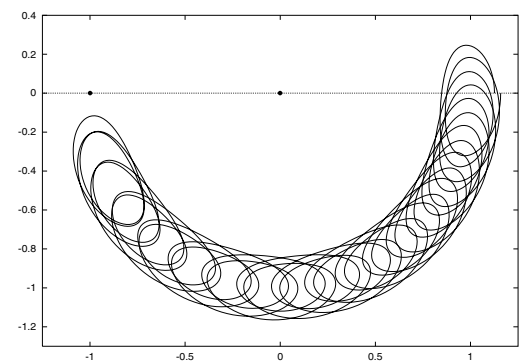

(3)

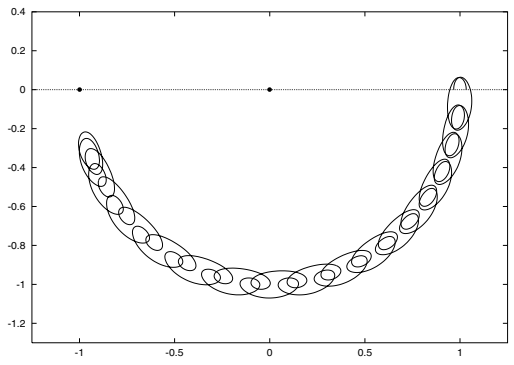

(6)

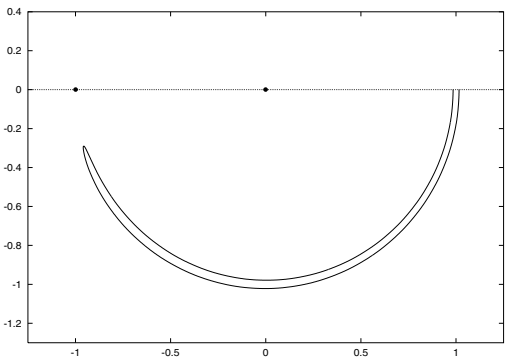

(9)

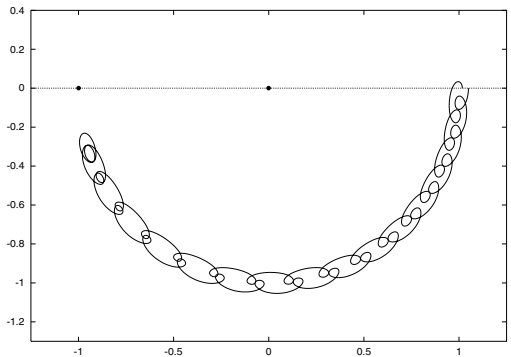

(12)

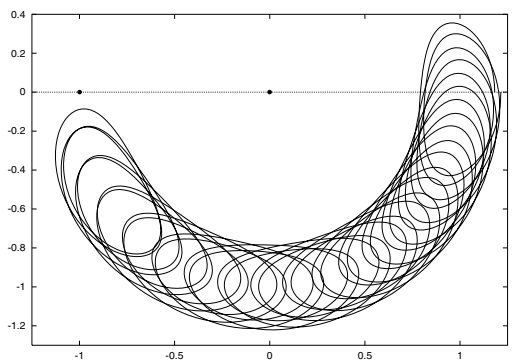

Fig. 13. Periodic horseshoe orbits from family A. On the top of each figure the number of the orbit, according to Table 2 , is given. For greater clarity, only the half period of each orbit is shown.

- there exists one orbit inside each family with a minimum value of the eccentricity. Starting at this orbit, the eccentricity increases along each branch. In general, the orbit with the minimum eccentricity and the orbit with the maximum Jacobi constant do not coincide;

- as the eccentricity increases, the width of the orbits increases;

- planar stable orbits with values of the Jacobi constant near $C_{\mathrm{Jm}}$ can be found. Far from this value, the planar stability parameter $s_{1}$ increases as $C_{\mathrm{J}}$ decreases.

And the differences are:

- the period $T$ of the orbits increases as $C_{\mathrm{Jm}}$ decreases;

- the width of the orbits increases as $C_{\mathrm{Jm}}$ increases;
- the bigger the value of $C_{\mathrm{Jm}}$, the faster the planar stability parameter $s_{1}$ increases.

\subsection{Stability}

Although we are dealing with planar periodic orbits, which are confined in the $x y$ plane, we will next consider not only planar perturbations but vertical ones as well (i.e. in the $z$ direction). Thus, we take into account the vertical component of the motion and write $\boldsymbol{q}\left(t, \boldsymbol{q}_{0}\right)=(x, y, z, \dot{x}, \dot{y}, \dot{z})$ for the position and velocity of the third body with initial conditions $\boldsymbol{q}_{0}$.

Let $\boldsymbol{q}\left(t, \boldsymbol{q}_{0}\right)$ be a periodic solution of period $T$ of the equations of motion (1). If we modify the initial conditions, then

$\boldsymbol{q}\left(T, \boldsymbol{q}_{0}+\Delta \boldsymbol{q}\right)=\boldsymbol{q}\left(T, \boldsymbol{q}_{0}\right)+M(T) \Delta \boldsymbol{q}+\ldots$ 
(1)

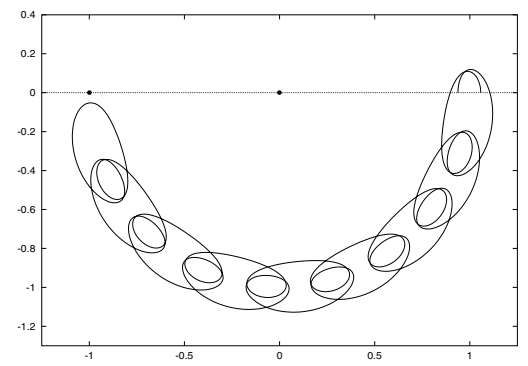

(4)

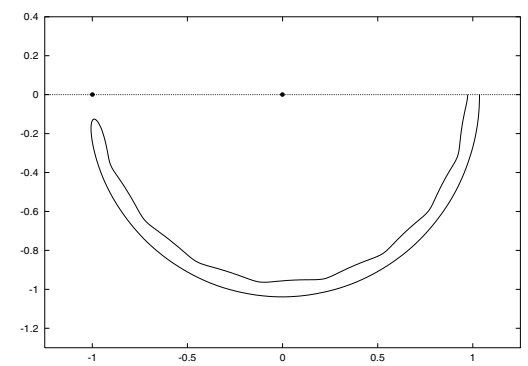

(7)

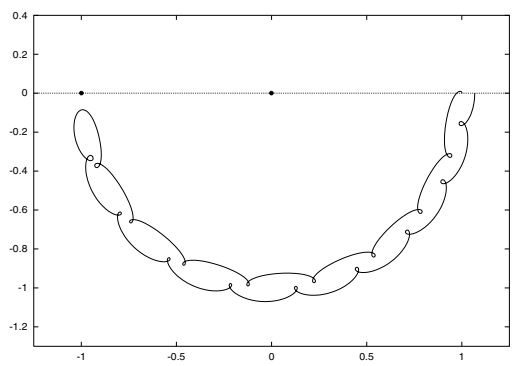

(2)

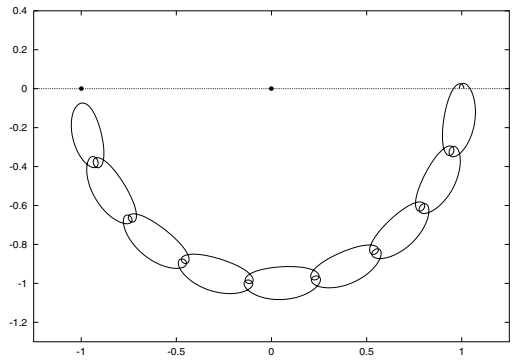

(5)

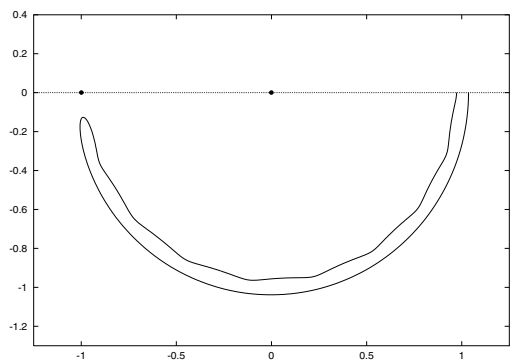

(8)

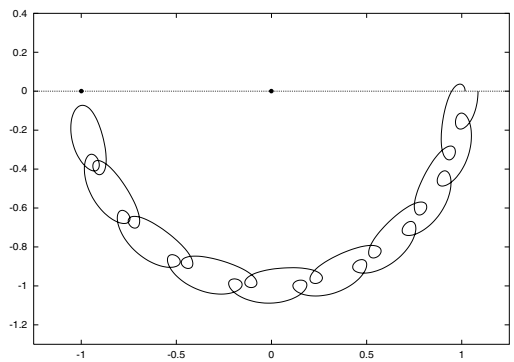

(3)

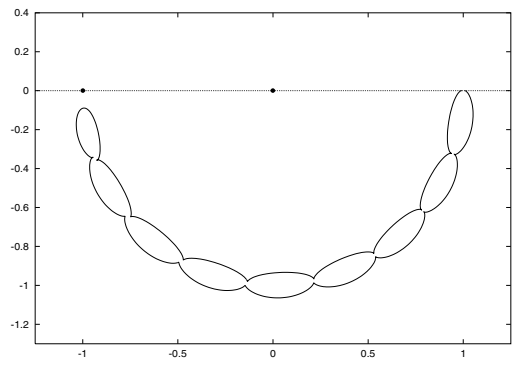

(6)

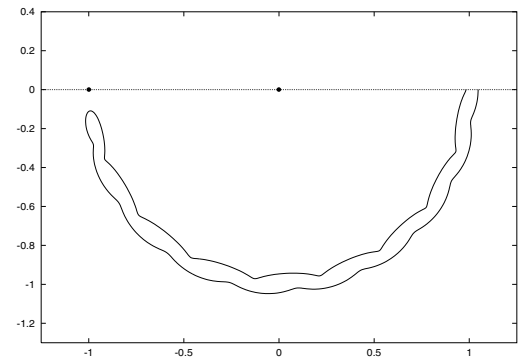

(9)

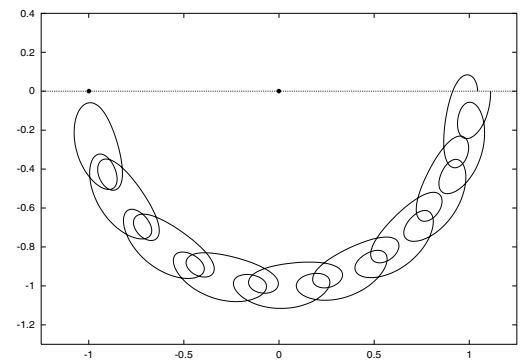

Fig. 14. Periodic horseshoe orbits from family B. On the top of each figure the number of the orbit, according to Table 2, is given. For greater clarity, only the half period of each orbit is shown.

(1)

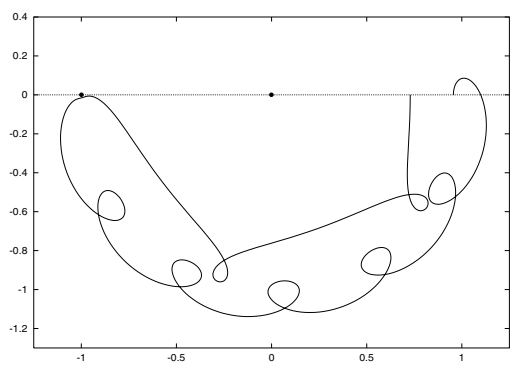

(4)

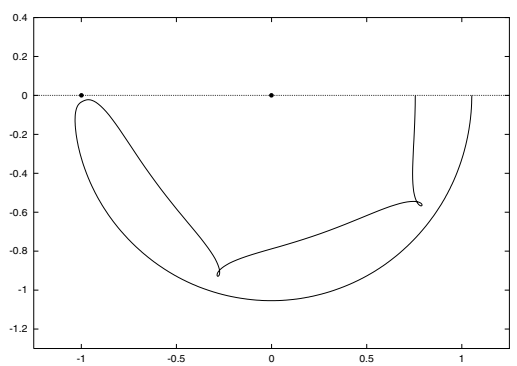

(2)

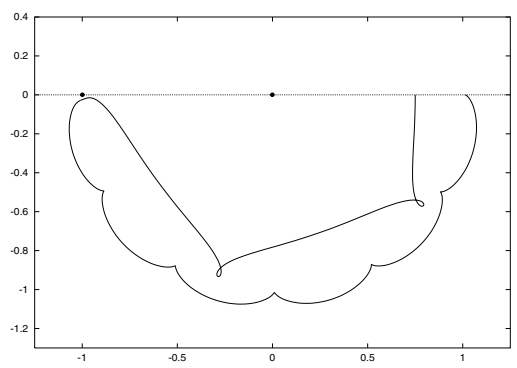

(5)

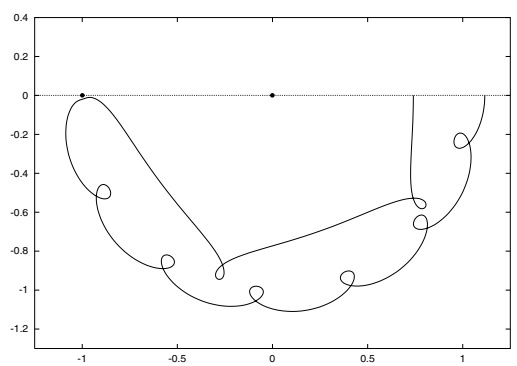

(3)

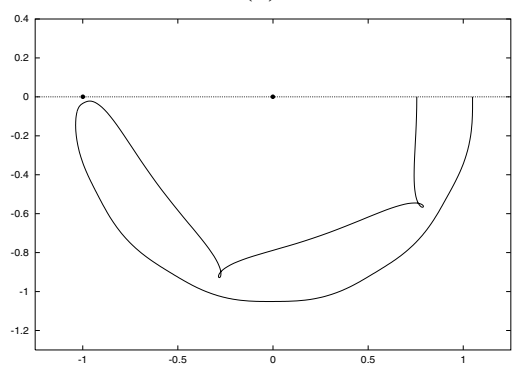

(6)

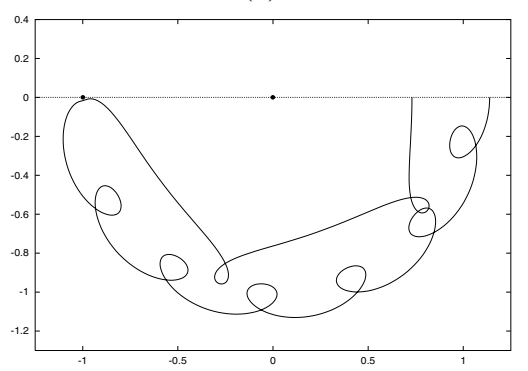

Fig. 15. Periodic horseshoe orbits from family C. On the top of each figure the number of the orbit, according to Table 2, is given. For greater clarity, only the half period of each orbit is shown. 
Table 2. For the families A, B and C, Jacobi constant, period, initial conditions and planar stability parameter of the orbits shown in Figs. 13-15. The orbits are identified with the label of the family (capital letter) and a number. For each family, the orbit with the minimum value of the eccentricity (*) and the orbit with maximum value of the Jacobi constant, $C_{\mathrm{Jm}}$ are included.

\begin{tabular}{|c|c|c|c|c|c|}
\hline 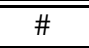 & 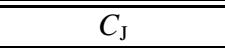 & $T / 2 \pi$ & $x_{0}$ & $\overline{\dot{y}_{0}}$ & $s_{1}$ \\
\hline $\mathrm{A}(1)$ & 2.978219881125 & 67.05634232 & 0.864394016091 & 0.288028401448 & 10.76794895771 \\
\hline $\mathrm{A}(2)$ & 2.994003281604 & 67.06797264 & 0.934493501386 & 0.140321084564 & 2.014742519509 \\
\hline $\mathrm{A}(3)$ & 2.998053265806 & 67.07241135 & 0.966643996979 & 0.074633914254 & 1.916231113201 \\
\hline $\mathrm{A}(4)$ & 2.999434544650 & 67.07415468 & 0.984418815860 & 0.038800214277 & 1.943509348146 \\
\hline $\mathrm{A}(5)$ & 3.000275933844 & 67.07527534 & 1.005383006601 & -0.003057364559 & 1.981234314265 \\
\hline $\mathrm{A}(6)^{*}$ & 3.000384180205 & 66.09063002 & 1.015982828023 & -0.023879698526 & 1.994651789393 \\
\hline $\mathrm{A}(7)$ & 3.000259851924 & 66.07701915 & 1.027126161963 & -0.045850645455 & 2.177762710756 \\
\hline $\mathrm{A}(8)$ & 2.999994017594 & 66.07657747 & 1.035799232525 & -0.062878546678 & 2.333101006854 \\
\hline $\mathrm{A}(9)$ & 2.999273436278 & 66.07555044 & 1.049466716047 & -0.089584341733 & 2.645571027888 \\
\hline $\mathrm{A}(10)$ & 2.995349095756 & 66.07075692 & 1.087211607215 & -0.162571013180 & 4.537180388699 \\
\hline $\mathrm{A}(11)$ & 2.980034257528 & 66.05835585 & 1.158570106019 & -0.297800763393 & 25.16722902490 \\
\hline $\mathrm{A}(12)$ & 2.960647051626 & 66.04910284 & 1.214480026998 & -0.401585865744 & 102.13641174368 \\
\hline $\mathrm{B}(1)$ & 2.992305408899 & 35.17287785 & 0.937751153239 & 0.141602106272 & $0.17667998 \mathrm{D}+05$ \\
\hline $\mathrm{B}(2)$ & 2.998927115544 & 35.26242610 & 0.987106194057 & 0.042181383629 & $0.46846892 \mathrm{D}+04$ \\
\hline $\mathrm{B}(3)$ & 3.000384482183 & 35.33885144 & 1.007927529189 & 0.001051532161 & $0.14763765 \mathrm{D}+04$ \\
\hline $\mathrm{B}(4)$ & 3.001100325914 & 35.73972610 & 1.035117446627 & -0.052032828838 & 1.998692428426 \\
\hline $\mathrm{B}(5)^{*}$ & 3.001100061306 & 35.76145941 & 1.035516752285 & -0.052814311462 & -0.116081477939 \\
\hline $\mathrm{B}(6)$ & 3.000844207702 & 36.35436782 & 1.046569507496 & -0.075220742861 & 165.20117846148 \\
\hline $\mathrm{B}(7)$ & 2.999808578648 & 36.27945671 & 1.067645762201 & -0.116293850771 & $0.17443203 \mathrm{D}+04$ \\
\hline $\mathrm{B}(8)$ & 2.998244463824 & 36.23273693 & 1.085457246038 & -0.150651378706 & $0.44575584 \mathrm{D}+04$ \\
\hline $\mathrm{B}(9)$ & 2.994673233458 & 36.18477531 & 1.112349859300 & -0.202041957868 & $0.10514385 \mathrm{D}+05$ \\
\hline $\mathrm{C}(1)$ & 2.993491365689 & 16.46863176 & 0.955652127541 & 0.113151877966 & $0.25144753 \mathrm{D}+06$ \\
\hline $\mathrm{C}(2)$ & 3.000768690758 & 16.68170901 & 1.013930358679 & -0.002175916753 & $0.79886505 \mathrm{D}+05$ \\
\hline $\mathrm{C}(3)$ & 3.002228501173 & 17.06721323 & 1.049004413169 & -0.070286026016 & -0.577877356154 \\
\hline $\mathrm{C}(4)^{*}$ & 3.002112554758 & 17.54131489 & 1.052217067968 & -0.077360889657 & $0.21503965 \mathrm{D}+04$ \\
\hline $\mathrm{C}(5)$ & 2.997662296979 & 17.53494048 & 1.115835035023 & -0.199952697571 & $0.14719198 \mathrm{D}+06$ \\
\hline $\mathrm{C}(6)$ & 2.994097444023 & 17.46506819 & 1.138790112334 & -0.243243615674 & $0.22424176 \mathrm{D}+06$ \\
\hline
\end{tabular}

where $M(t)$ is the monodromy matrix at time $t$. In order to study the linear stability of the periodic orbits, we have to look at the eigenvalues of the matrix $M(T)$. As the motion is planar, we know that the monodromy matrix is

$M(t)=\left(\begin{array}{cccccc}m_{11} & m_{12} & 0 & m_{13} & m_{14} & 0 \\ m_{21} & m_{22} & 0 & m_{23} & m_{24} & 0 \\ 0 & 0 & a_{\mathrm{v}} & 0 & 0 & b_{\mathrm{v}} \\ m_{31} & m_{32} & 0 & m_{33} & m_{34} & 0 \\ m_{41} & m_{42} & 0 & m_{43} & m_{44} & 0 \\ 0 & 0 & c_{\mathrm{v}} & 0 & 0 & d_{\mathrm{v}}\end{array}\right)$

which can be decoupled into two matrices,

$M_{\mathrm{p}}(t)=\left(\begin{array}{llll}m_{11} & m_{12} & m_{13} & m_{14} \\ m_{21} & m_{22} & m_{23} & m_{24} \\ m_{31} & m_{32} & m_{33} & m_{34} \\ m_{41} & m_{42} & m_{43} & m_{44}\end{array}\right), \quad M_{\mathrm{v}}(t)=\left(\begin{array}{ll}a_{\mathrm{v}} & b_{\mathrm{v}} \\ c_{\mathrm{v}} & d_{\mathrm{v}}\end{array}\right)$,

called the planar monodromy matrix and vertical monodromy matrix, respectively. For simplicity, we denote $M_{\mathrm{p}}=M_{\mathrm{p}}(T)$ and $M_{\mathrm{v}}=M_{\mathrm{v}}(T)$. Known properties include:

1. $\operatorname{det}\left(M_{\mathrm{p}}\right)=\operatorname{det}\left(M_{\mathrm{v}}\right)=1$;

2. the eigenvalues of $M(T)$ are $1,1, \lambda_{1}, 1 / \lambda_{1}, \lambda_{2}, 1 / \lambda_{2}$. In fact, for planar orbits, $1,1, \lambda_{1}, 1 / \lambda_{1}$ are eigenvalues of $M_{\mathrm{p}}$ and $\lambda_{2}, 1 / \lambda_{2}$ are eigenvalues of $M_{\mathrm{v}}$;
3. the stability parameters are defined as $s_{1}=\lambda_{1}+1 / \lambda_{1}$ and $s_{2}=\lambda_{2}+1 / \lambda_{2}$. For planar orbits, $s_{1}$ and $s_{2}$ are the planar and vertical stability parameter respectively;

4. $s_{1}=2-\operatorname{tr}\left(M_{\mathrm{p}}\right)$ and $s_{2}=\operatorname{tr}\left(M_{\mathrm{v}}\right)$, where $\operatorname{tr}(M)$ means the trace of the matrix $M$;

5. for planar symmetric orbits $a_{\mathrm{v}}=d_{\mathrm{v}}$ (see, for instance, Henon 1973).

A periodic orbit is planar or vertically stable if $\left|s_{1}\right|<2$ or $\left|s_{2}\right|<2$ respectively. If both values are between -2 and 2 , then we will simply say that the orbit is (linearly) stable. If the stability parameter equals 2 or -2 , the orbit is critical.

For each planar horseshoe orbit, we compute both stability parameters. We are interested in vertically stable or critical orbits because this is where bifurcations can occur, but also where the planar stable orbits are located. In Fig. 16, the zones of planar stability of each family are shown.

From the results obtained, we observe that:

1. the stability parameter $s_{1}$ varies from positive values to negative ones covering the range $[-2,2]$ a least twice. Given a family, the smaller the value of $C_{\mathrm{Jm}}$, the bigger the zones on the characteristic curves corresponding to planar stable orbits. The families with $C_{\mathrm{Jm}}>3.0013$ are not shown in Fig. 16 because the zones of planar stable orbits cannot be observed. Nevertheless, all families have a least one zone of planar stable orbits; 


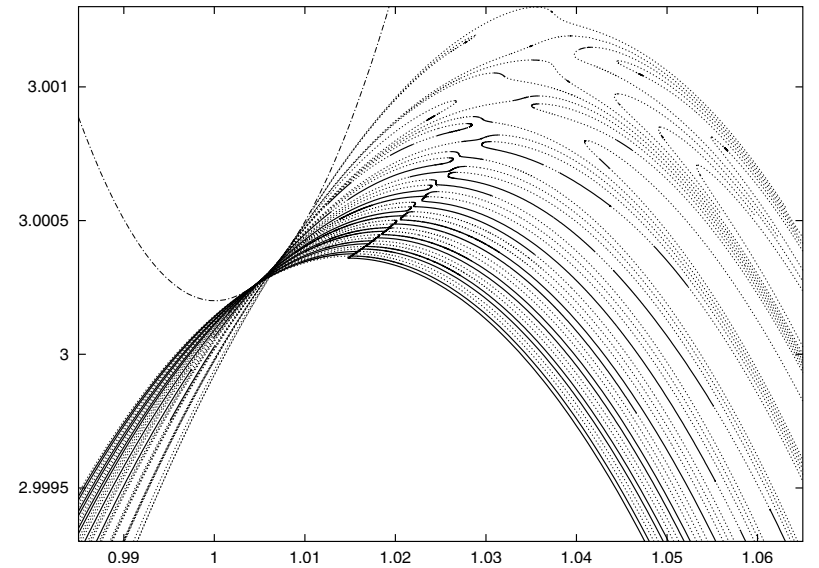

Fig. 16. Planar stability in families of symmetric planar horseshoe orbits in the $\left(x_{0}, C_{\mathrm{J}}\right)$ plane. The zones corresponding to stable and unstable orbits are plotted in continuous and dotted lines respectively.

2. $s_{1}$ grows in both branches of each family, while $s_{2}$ seems to remain bounded. See Fig. 17;

3 . most orbits are vertically stable. There are vertically unstable orbits in some families with values of $C_{\mathrm{Jm}}$ greater than 3.0009364257. For $C_{\mathrm{Jm}}<3.0009364257$ no vertically unstable orbits are founded.

\section{Bifurcating 3D families}

The next step is to find the bifurcation orbits from which families of three dimensional orbits are born and compute them.

A $3 \mathrm{D}$ family is born from a perturbation of the $z$ and $\dot{z}$ components of a planar periodic orbit. If the perturbation is small enough, the motion of the third particle can be decoupled in a planar motion plus a vertical one in a first approximation. If we substitute $z$ by $\varepsilon z$, on the Eqs. (1), we obtain

$\ddot{x}-2 \dot{y}=\frac{\partial \Omega}{\partial x}(x, y, 0)+O(\varepsilon)$,

$\ddot{y}+2 \dot{x}=\frac{\partial \Omega}{\partial y}(x, y, 0)+O(\varepsilon)$,

$\ddot{z}=-z\left(\frac{1-\mu}{r_{1}(x, y, 0)^{3}}+\frac{\mu}{r_{2}(x, y, 0)^{3}}\right)+O(\varepsilon)$.

Setting $\varepsilon=0$ in Eq. (8), we obtain two decoupled motions where the first two equations correspond to the planar RTBP. Thus, a periodic solution of Eq. (8) for $\varepsilon=0$ will be a periodic solution of the planar RTBP plus a periodic motion in the vertical direction given by the solution of the equation

$\ddot{z}+g(t) z=0$.

We are looking for 3D-periodic orbits, so both the planar and the vertical motion must have the same period. Let $\boldsymbol{r}=(x, y, z)$ be the solution of the Eqs. (8) for $\varepsilon=0$, with initial conditions $\boldsymbol{r}_{0}=\left(x_{0}, y_{0}, z_{0}\right)$ and $\dot{\boldsymbol{r}}_{0}=\left(\dot{x}_{0}, \dot{y}_{0}, \dot{z}_{0}\right)$ such that $\left(x_{0}, y_{0}\right)$ and $\left(\dot{x}_{0}, \dot{y}_{0}\right)$ are the initial conditions of a planar $T$-periodic orbit. Then, both the planar motion $(x(t), y(t))$ and the function $g(t)$ are $T$-periodic. Applying Floquet's theorem (see, for example, Boccaletti \& Pucacco 1999, p. 199) to the Eq. (9) we get

$\Phi(t)=P(t) \exp (R t)$,
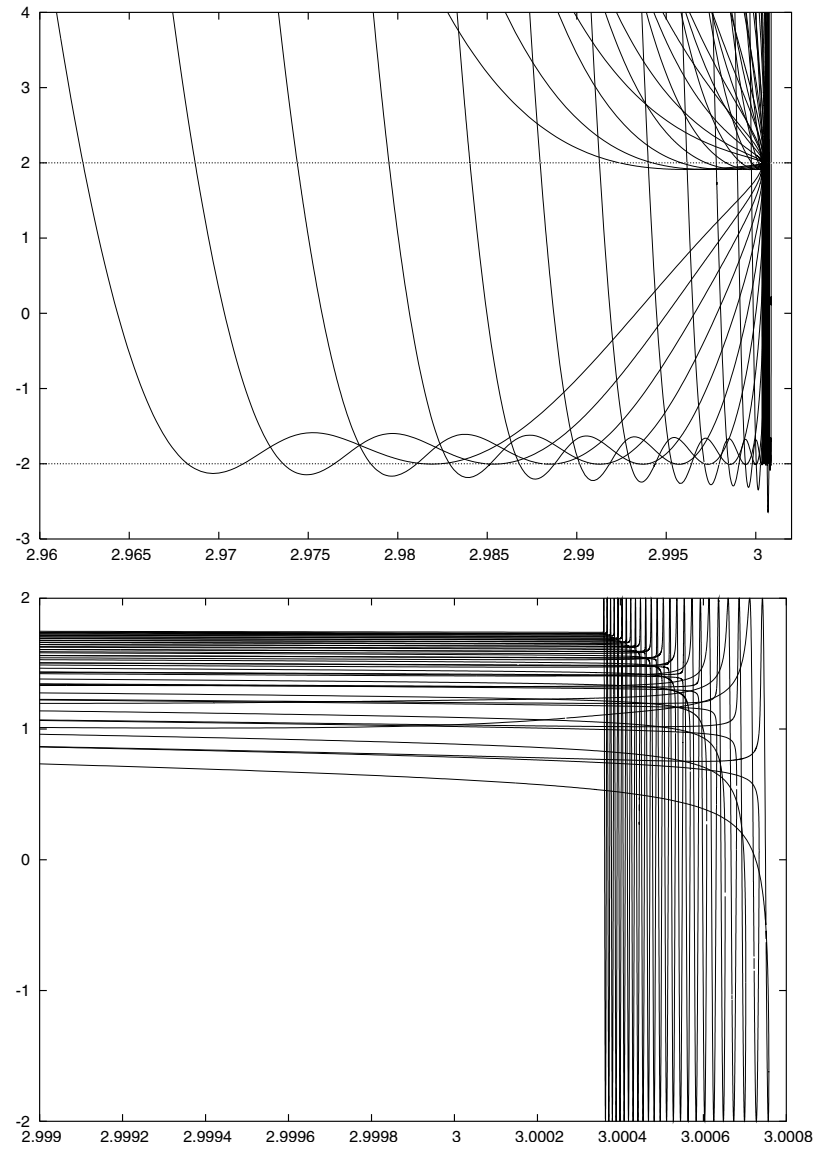

Fig. 17. The stability parameters (on the vertical axis) versus the Jacobi constant (on the horizontal one) are represented for some families of horseshoe orbits: on the top, the planar stability parameter $s_{1}$, and on the bottom, the vertical one $s_{2}$.

where $\Phi(t)$ is the fundamental matrix, $P(t)$ is a continuous $T$-periodic matrix and $R$ is a constant matrix. This implies that the solutions of Eq. (9) can be written as

$z(t)=c_{1} \mathrm{e}^{\rho t} P_{1}(t)+c_{2} \mathrm{e}^{-\rho t} P_{2}(t)$,

where $P_{i}(t), i=1,2$, are $T$-periodic and $\rho$ and $-\rho$ are the eigenvalues of $R$. Moreover, $\Phi(t)=M_{\mathrm{v}}$, the vertical monodromy matrix of the planar orbit. Using this fact and Eq. (10), we get

$\rho=\frac{1}{T} \ln \left(\lambda_{2}\right)$

where $\lambda_{2}$ and $1 / \lambda_{2}$ are the eigenvalues of $M_{\mathrm{v}}$.

Floquet's theorem tells when $z(t)$ given by Eq. (11) is periodic. The vertical motion is bounded if $\rho=i v$ and, in this case, the two periods involved are $T$ and $2 \pi / v$. For periodicity of the $3 \mathrm{D}$ motion these periods must satisfy the condition $p T=q 2 \pi / v$ for some mutually prime positive integers $p$ and $q$, or equivalently

$\theta=v T=2 \pi \frac{q}{p}$.

Using Eqs. (12), (13) and the definition of the vertical stability parameter, we can write

$s_{2}=\mathrm{e}^{i v T}+\mathrm{e}^{-i v T}=2 \cos (\theta)$. 
Thus, if the planar orbit is vertically stable and Eq. (14) is verified, $\boldsymbol{r}=(x, y, z)$ is a periodic solution of period $p T$. From this we know which planar orbits will be bifurcation orbits.

Critical orbits are bifurcation orbits as well. Using the linear approximation given by the monodromy matrix $M_{\mathrm{v}}$, Henon (1973) has shown that critical orbits of the main families of periodic orbits a, b, c, f, g, h, i, l, m, n (in Strömgren's notation) correspond to an intersection of a planar family and a spatial one. If $s_{2}=2$, the spatial orbit has the same period as the planar one, and if $s_{2}=-2$ the spatial orbit doubles the period of the planar one. From now on, a bifurcation orbit with vertical stability parameter $s_{2}=2 \cos (2 \pi q / p)$ will be called a $(p, q)$-bifurcation orbit, or briefly, $p$-bifurcation orbit if we do not care about the value of $q$. Notice that, for every value of $p$, both $q$ and $p-q$ give the same value of $s_{2}$. Then, given a value of $p$, we will only consider the values of $q \in\{1,2, \ldots,[p / 2]\}$ such that $p$ and $q$ are relatively prime ([x] means the integer part of $x$ ).

For $\varepsilon>0$, this reasoning tells us which planar orbits are good to begin with. In the next section, we explain the steps that we followed.

\subsection{Generating spatial horseshoe orbits}

As in the planar case, we use a predictor-corrector method to compute the orbits embedded in the same family. On the one hand, it is necessary to provide the equations that define the families of 3D-periodic orbits. On the other hand, as a starting point of the method, we take a planar $T$-periodic orbit satisfying Eqs. (13) and (14) and we modify slightly the initial conditions in the vertical direction. These initial conditions must then be corrected to have a $3 \mathrm{D}$ periodic orbit.

We restrict our attention to symmetric orbits. In the three dimensional problem, the equations of the RTBP satisfy the following symmetries:

$$
\begin{aligned}
& (t, x, y, z, \dot{x}, \dot{y}, \dot{z}) \longrightarrow(-t, x,-y, z,-\dot{x}, \dot{y},-\dot{z}), \\
& (t, x, y, z, \dot{x}, \dot{y}, \dot{z}) \longrightarrow(-t, x,-y,-z,-\dot{x}, \dot{y}, \dot{z}) .
\end{aligned}
$$

It follows that if $\boldsymbol{q}(t)$ is a solution of Eq. (1), such that

$$
\boldsymbol{q}(0)=\left(x_{0}, 0, z_{0}, 0, \dot{y}_{0}, 0\right) \text { and } \boldsymbol{q}(\bar{T} / 2)=\left(x_{1}, 0, z_{1}, 0, \dot{y}_{1}, 0\right)
$$

or

$$
\boldsymbol{q}(0)=\left(x_{0}, 0,0,0, \dot{y}_{0}, \dot{z}_{0}\right) \text { and } \boldsymbol{q}(\bar{T} / 2)=\left(x_{1}, 0,0,0, \dot{y}_{1}, \dot{z}_{1}\right),
$$

the orbit is $\bar{T}$-periodic. If Eq. (15) is satisfied, the orbit is symmetric with respect to the $y=0$ plane, and if Eq. (16) is satisfied, then it is symmetric with respect to the $z=0$ and $y=0$ planes.

Let us suppose that we have a planar and stable symmetric $p$-bifurcation orbit of period $T$ and initial conditions $\left(x_{0}, 0,0,0, \dot{y}_{0}, 0\right)$. Let $v=\left(\Delta z_{0}, \Delta \dot{z}_{0}\right)$ be a small perturbation in the vertical direction. Keeping in mind that we are looking for a 3D periodic orbit of period $\bar{T}=p T$, we consider again the motion as two decoupled motions, thus the planar one is already symmetric. Considering the vertical motion, we use the linear approximation given by the monodromy matrix $M_{\mathrm{v}}(t)$. Thus

$v_{1}=M_{\mathrm{v}}(p T / 2) v_{0}$,
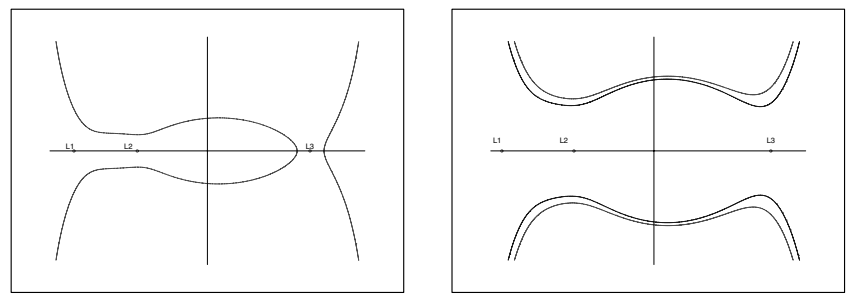

Fig. 18. The intersection of the zero velocity surfaces with the $y=0$ plane: a) for $C_{\mathrm{J}} \in\left[C_{\mathrm{J}_{3}}, C_{\mathrm{J}_{1}}\right]$; and $\left.\mathbf{b}\right)$ for $C_{\mathrm{J}}<C_{\mathrm{J}_{3}}$.

where $v_{1}$ is the vertical perturbation at time $t=p T / 2$. The properties of $M_{\mathrm{v}}$ tell us that it has the form

$M_{\mathrm{v}}=M_{\mathrm{v}}(T)=\left(\begin{array}{cc}\cos \theta & b \\ -\frac{\sin ^{2} \theta}{b} & \cos \theta\end{array}\right)$,

with some constant $b$. If $p=2 m$, then $M_{\mathrm{v}}(p T / 2)=M_{\mathrm{v}}^{m}=-I$ $\left(I=\right.$ identity matrix). For an initial perturbation $v_{0}=\left(\Delta z_{0}, 0\right)$, we have $v_{1}=\left(\Delta z_{1}, 0\right)$, in agreement with Eq. (15). Similarly, for $v_{0}=\left(0, \Delta \dot{z}_{0}\right), v_{1}=\left(0, \Delta \dot{z}_{1}\right)$, which agrees with Eq. (16). In both cases we obtain a symmetric periodic orbit. The same happens when $p=2 m+1$, since then

$M_{\mathrm{v}}(p T / 2)=M_{\mathrm{v}}(T / 2) M_{\mathrm{v}}^{m}=\left(\begin{array}{cc}\alpha & 0 \\ 0 & 1 / \alpha\end{array}\right)$,

for some $\alpha \neq 0$. (For the relation between the matrix $M_{\mathrm{v}}(T / 2)$ and $M_{\mathrm{v}}(T)$ see Henon 1973.)

Thus the symmetric $p$-bifurcation orbits are bifurcation orbits from which families of symmetric periodic 3D orbits arise. If we take one of them and introduce a small perturbation in the $z$ or $\dot{z}$ direction, we can expect to find symmetric and periodic spatial orbits. Initially, the period will be approximately $p T$ whereas, as the family is followed, the period will change.

We proceed as follows:

1. first, we modify slightly the initial conditions $z_{0}$ or $\dot{z}_{0}$ (not both at the same time) in order to have initial conditions like in Eqs. (15) or (16). For both symmetries, the orbit starts off perpendicular to the plane $y=0$ and at half period it again crosses the same section perpendicularly. If there are only two crossings of the plane $y=0$ per period, the total number of crossings is $2 p$ (since the period of the spatial orbit is $=p T)$, so in a half a period it is $p$. In the case of more crossings these numbers are increased accordingly;

2. if the initial perturbation is in the $z$ direction, then by Eq. (15), the equations defining the periodic orbits are

$$
\dot{x}_{\mathrm{f}}(t)=0, \quad \dot{z}_{\mathrm{f}}(t)=0,
$$

where $t$ is defined implicitly by the condition $y(t)=0$ at the $p$ th time when the orbit crosses the plane $y=0$. If the initial perturbation is in the $\dot{z}$ direction, using Eq. (16), the equations are

$$
\dot{x}_{\mathrm{f}}(t)=0, \quad z_{\mathrm{f}}(t)=0 .
$$

These equations enable us to correct the starting point and predict a new one, using the tangent space to the manifold defined 

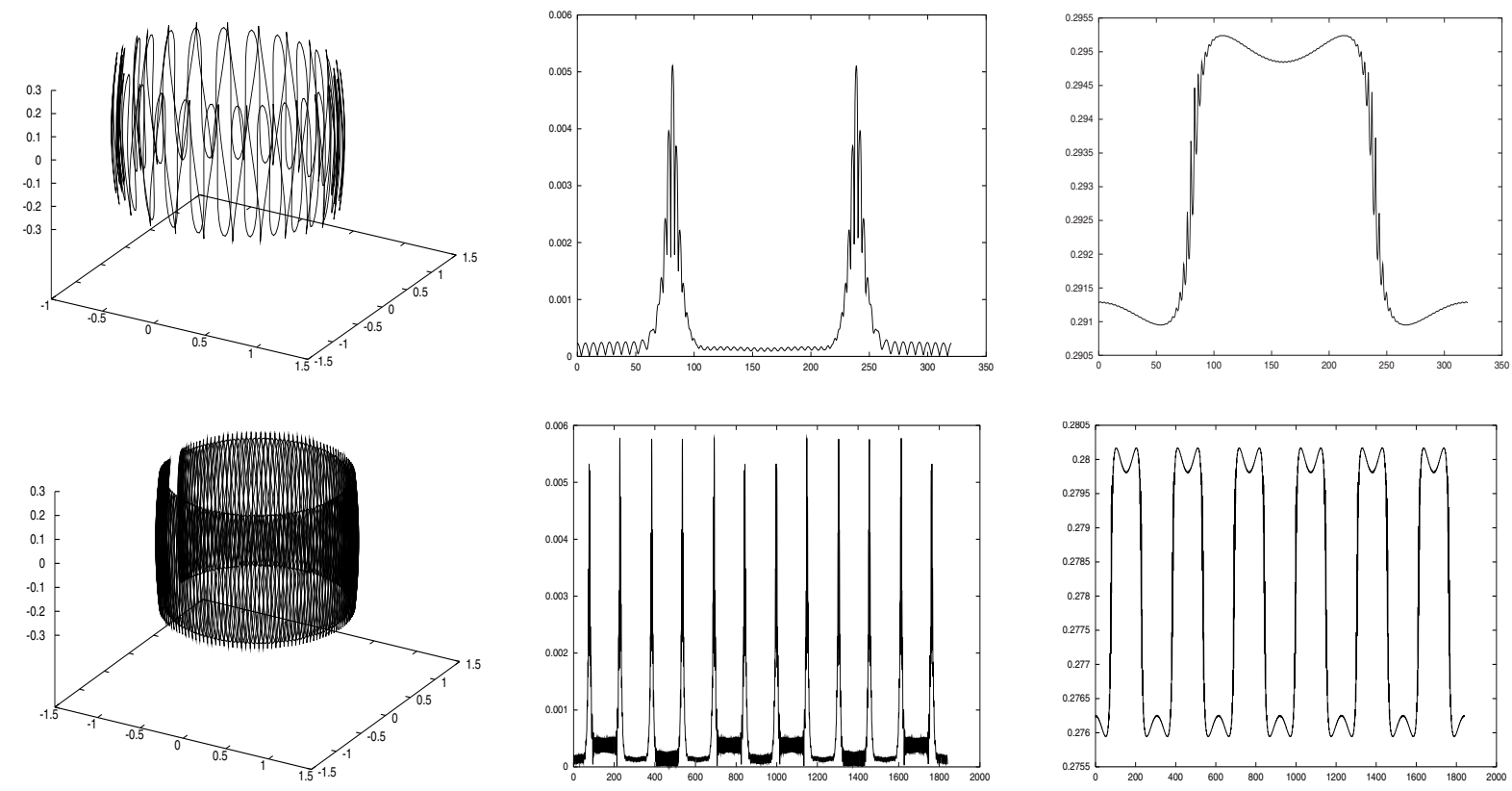

Fig. 19. Examples of 3D periodic horseshoe orbits. On the top, an orbit of a family of type $1 / 1$ and on the bottom, an orbit of a family of type 6/2. The figures in the center and right show the behavior of the eccentricity and the inclination respectively, both along one period. The horseshoe orbital behavior can be observed in the changes of the eccentricity and inclination every time the third body approaches the small primary.

Table 3. Values of $p$ and $s_{2}$ of the bifurcation orbits used.

\begin{tabular}{cccccc}
\hline \hline$p$ & 1 & 2 & 3 & 4 & 6 \\
\hline$s_{2}$ & 2 & -2 & -1 & 0 & 1 \\
\hline
\end{tabular}

by them. Note that every initial planar stable periodic orbit generates two families of spatial orbits. This is not true in general for critical orbits ( $p=1,2)$, but from the results obtained, we see that from every critical orbit of the horseshoe families computed in the previous sections, two families of spatial orbits arise.

\subsection{Families of spatial horseshoe orbits}

Given fixed values $p$ and $q$, we take a stable planar $p$-bifurcation orbit. Such an orbit always exists inside families with $C_{\mathrm{J}_{\mathrm{m}}}$ less than a certain value. For $\mu=10^{-4}$ :

- in each family with $C_{\mathrm{Jm}}<3.0008627$, for any value $\sigma \in$ $(-2,2)$ there exist at least two stable orbits with $s_{2}=\sigma$;

- in each family with higher values of $C_{\mathrm{Jm}}$, there exist values $\sigma \in(-2,2)$ such that no stable orbit has $s_{2}=\sigma$.

We followed some spatial families for the values of $p$ and $s_{2}$ given in Table 3 .

As we have already said, from each bifurcation orbit two families of spatial orbits arise. Orbits with initial conditions $\left(x_{0}, z_{0}, \dot{y}_{0}\right)$ are called of type 1 , and orbits with initial conditions $\left(x_{0}, \dot{y}_{0}, \dot{z}_{0}\right)$, of type 2 (the other initial coordinates are zero). We say that a family is of type $p / i$ if it bifurcates from a $p$-bifurcation orbit and its orbits are of type $i$. In Fig. 19 two spatial periodic horseshoe orbits and their eccentricity and inclination are shown. As we can observe, the inclination oscillates around a mean value.

From the results obtained we observe the following:

1. For simplicity, we consider the inclination of one orbit as its value at the initial condition, instead of its average value. The inclination will be given by

$\cos i=\frac{x_{0}}{\sqrt{x_{0}^{2}+z_{0}^{2}}}, \quad$ or $\quad \cos i=\frac{x_{0}+\dot{y}_{0}}{\sqrt{\left(x_{0}+\dot{y}_{0}\right)^{2}+\dot{z}_{0}^{2}}}$

for orbits of type 1 and 2 respectively. In each family, in general, as the Jacobi constant decreases, the inclination increases. Nevertheless, we found some families where the inclination increases until a maximum value and then decreases. See Fig. 20.

2. Within each family, the eccentricity (at the initial conditions) increases and the minimum distance to the small primary always decreases. This means, as in the planar case, that the number of loops increases and that the orbits briefly enter the half-space $y>0$.

3. For a fixed value of the Jacobi constant, there is a maximum possible inclination given by the zero velocity surfaces. In the planar case, there are no zero velocity curves for $C_{\mathrm{J}} \leq 3-\mu(1-\mu)$, but in the spatial case there are forbidden regions for the motion for $C_{\mathrm{J}}>-\mu(1-\mu)$, although they do not intersect the $z=0$ plane. See Fig. 18. As the 

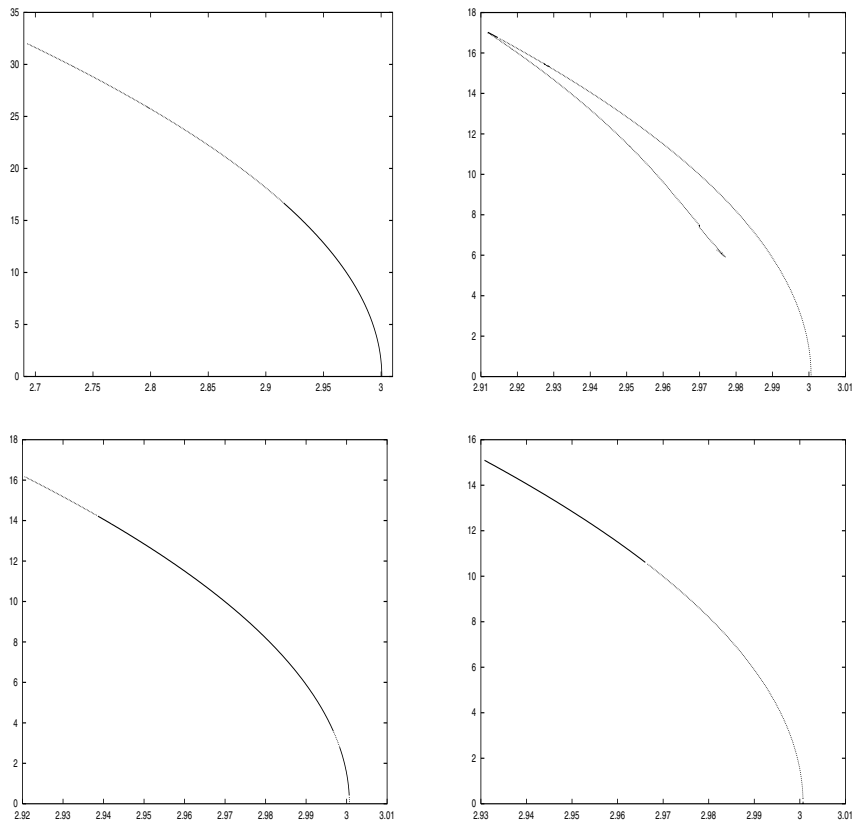

Fig. 20. Jacobi constant versus inclination (in degrees) of four different families of spatial horseshoe orbits. The stability zones are plotted in continuous and dotted lines respectively. From left to right and top to bottom, the families are of type: a) $1 / 1$; b) $1 / 2$; c) $3 / 1$; d) $3 / 2$.

initial conditions are near the $z v s$, the height of the orbit is almost as larger as it can be, and so is the inclination.

4. Concerning stability, no simple rule seems to exist. Each family may or may not have stable orbits. In general, a larger inclination seems to imply stronger instability. When a family contains stable orbits, there is a "critical" inclination separating stable and unstable orbits (Figs. 20 and 21). For $\mu=10^{-4}$, we find stable orbits up to inclinations of $17^{\circ}$.

\section{Conclusions}

In this paper we have computed families of planar and spatial symmetric periodic horseshoe orbits.

First we described the organization and stability of planar orbits. This allowed us to find bifurcation orbits and to compute families of three-dimensional horseshoe orbits, as well.

The values of the Jacobi constant, stability parameter, eccentricity and inclination of these orbits were outlined. We found that spatial stable orbits up to an inclination of $17^{\circ} \mathrm{ex}-$ ist. Our study does not exclude the possible existence of stable periodic orbits of even higher inclination. This remains to be investigated.

Acknowledgements. The first author acknowledges the Tuorla Observatory where most of this work has been carried out, and is partially supported by the Catalan grant CIRIT 2001SGR-00070 and Spanish grant DGICYT BFM2003-09504-C02-01.
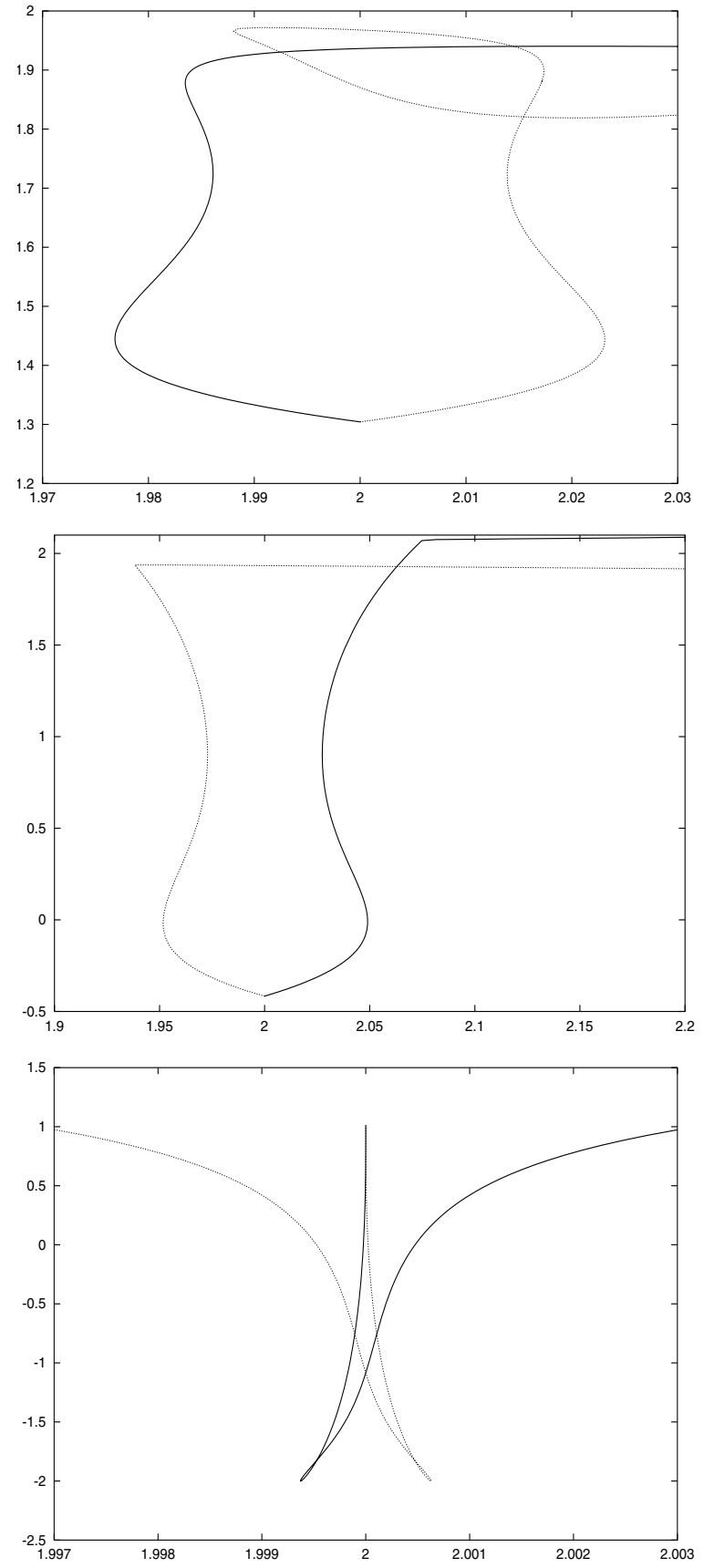

Fig. 21. Behaviour of the stability parameters $\left(s_{1}\right.$ on the horizontal axis and $s_{2}$ on the vertical one) for families of type $p / 1$ (continuous line) and $p / 2$ (dotted line) for different values of $p$. a) $p=1$; b) $p=2$; and c) $p=3$.

\section{References}

Boccaletti, D., \& Pucacco, G. 1999, Theory of Orbits, 2: Perturbative and Geometrical Methods (Berlin: Springer-Verlag)

Brasser, R., Innanen, K. A., Connors, H., et al. 2004, Icarus, in press Burrau, C., \& Strömgren, E. 1915, Astron. Nachr., 200, 313

Burrau, C., \& Strömgren, E. 1916, Astron. Nachr., 202, 185

Burrau, C., \& Strömgren, E. 1917, Astron. Nachr., 203, 277

Christou, A. 2000, Icarus, 144, 1

Connors M., Chodas, P., Mikkola, S., et al. 2002, Meteoritics \& Planetary Science, 37, 1435 
Cors, J. M., \& Hall, G. R. 2003, SIAM J. Appl. Dyn. Syst., 2, 219

Dermott, S. F., \& Murray, C. D. 1981a, Icarus, 1

Dermott, S. F., \& Murray, C. D. 1981b, Icarus, 12

Gómez, G., \& Mondelo, J. 2001, Physica D, 157, 283

Henon, M. 1973, A\&A, 28, 415

Hénon, M. 1997, Generating families in the restricted three-body problem (Berlin: Springer-Verlag), 278

Hénon, M., \& Petit, J. M. 1986, Cel. Mech., 38, 67

Jorba, A., \& Zou, M., preprint

Llibre, J., \& Ollé, M. 2001, A\&A, 378, 1087

Namouni, F. 1999, Icarus, 137, 293
Namouni, F., Cristou, A. A., \& Murray, C. D. 1999, Phys. Rev. Lett., 83,2506

Murray, C. D., \& Dermott, S. F. 1999, Solar system dynamics (Cambridge: Cambridge University Press), 592

Salo, H., \& Yoder, F. 1988, A\&A, 309

Spirig, F., \& Waldvogel, J. 1985, in Stability of the Solar System and its minor natural and artificial satellites (D. Reidel Publishing Company), 53

Szebehely, V. 1967, Theory of Orbits. The Restricted Problem of Three Bodies (Academic Press, Inc.)

Wiegert, P., Innanen, K., \& Mikkola, S. 1997, Nature, 387, 685 